TRANSACTIONS OF THE

AMERICAN MATHEMATICAL SOCIETY

Volume 353, Number 1, Pages 327-347

S 0002-9947(00)02656-8

Article electronically published on September 13, 2000

\title{
CONVERGENCE OF THE RUELLE OPERATOR FOR A FUNCTION SATISFYING BOWEN'S CONDITION
}

\author{
PETER WALTERS
}

\begin{abstract}
We consider a positively expansive local homeomorphism $T: X \rightarrow$ $X$ satisfying a weak specification property and study the Ruelle operator $\mathcal{L}_{\varphi}$ of a real-valued continuous function $\varphi$ satisfying a property we call Bowen's condition. We study convergence properties of the iterates $\mathcal{L}_{\varphi}^{n}$ and relate them to the theory of equilibrium states.
\end{abstract}

\section{INTRODUCTION}

We consider a continuous map $T: X \rightarrow X$ of a compact metric space and a continuous function $\varphi: X \rightarrow \mathbb{R}$ with some assumptions that ensure the Ruelle operator $\mathcal{L}_{\varphi}$ maps the space $C(X ; \mathbb{R})$ of real-valued continuous functions to itself and behaves well. We obtain a convergence theorem for $\mathcal{L}_{\varphi}^{n}$ as $n \rightarrow \infty$ (Theorem 2.16) and deduce results about equilibrium states. Results of this type are mostly stated when $T$ is a subshift of finite type, but we use a more general context to include expanding maps of compact manifolds and other examples.

Let $X$ be a compact metric space with metric $d$, and let $T: X \rightarrow X$ be a continuous surjection. We shall assume $T$ is positively expansive, i.e. $\exists \delta_{0}>0$, so that if $x \neq z \exists n \geq 0$ with $d\left(T^{n} x, T^{n} z\right)>\delta_{0}$. Such a number $\delta_{0}$ is called an expansive constant for $T$ and clearly every smaller positive number is also an expansive constant. If we change to an equivalent metric, then $T$ is positively expansive in the new metric, but the expansive constant can change. Reddy has shown one can find an equivalent metric $D$ and constants $\tau>0, \lambda>1$ such that $D(x, z)<\tau$ implies $D(T x, T z) \geq \lambda D(x, z)$. Hence $T$ expands distances locally in the metric $D$ ( $\mathrm{Re})$.

We also assume $T$ is a local homeomorphism. This condition can be stated in several equivalent ways, which we discuss later in this section. The third assumption on $T$ is a weak specification condition, which can also be described in several ways (see Theorem 1.2). This condition does not involve periodic points. Topologically mixing subshifts of finite type are examples of positively expansive local homeomorphisms with the weak specification property, and they are the only subshifts with these properties. Another important class of examples is given by expanding differentiable maps of smooth compact connected manifolds.

We have assumed $T$ is a local homeomorphism because we want the transfer operators, defined by Ruelle, to map the Banach space $C(X ; \mathbb{R})$ of real-valued continuous functions on $X$, equipped with the supremum norm, to itself. For

Received by the editors August 9, 1999.

2000 Mathematics Subject Classification. Primary 37D35; Secondary 28D20, 37A30, 37B10.

Key words and phrases. Transfer operator, equilibrium state, entropy. 
each $\varphi \in C(X ; \mathbb{R})$ the transfer operator $\mathcal{L}_{\varphi}: C(X ; \mathbb{R}) \rightarrow C(X ; \mathbb{R})$ is defined by $\left(\mathcal{L}_{\varphi} f\right)(x)=\sum_{y \in T^{-1} x} e^{\varphi(y)} f(y)$. This is a finite sum; since $T$ is positively expansive, each set $T^{-1} x$ is $\delta_{0}$-separated if $\delta_{0}$ is an expansive constant. Each operator $\mathcal{L}_{\varphi}$ is linear, continuous and positive.

We take the opportunity to introduce some notation. We use 1 to denote the constant function with value 1 . The open ball with center $x$ and radius $\delta$ will be denoted by $B(x ; \delta)$. The $\sigma$-algebra of Borel subsets of $X$ will be denoted by $\mathcal{B}(X)$ or by $\mathcal{B}$ if no confusion can arise. The convex set, $M(X)$, of all probability measures on $(X, \mathcal{B})$ can be considered as a subset of the dual space $C(X ; \mathbb{R})^{*}$ and $M(X)$ is compact in the weak*-topology. The space of $T$-invariant members of $M(X)$ is also compact in the weak*-topology and is denoted by $M(X, T)$. If $\varphi \in C(X ; \mathbb{R})$, then $P(T, \varphi)$ denotes the pressure of $T$ at $\varphi([\mathrm{W} 2])$. If $\mu \in M(X)$, then $L_{\mu}^{p}(X)$ denotes the space of measurable $f: X \rightarrow \mathbb{R}$ with $|f|^{p}$ integrable with respect to $\mu, p \geq 1$. The conditional expectation of $f: X \rightarrow \mathbb{R}$ with respect to a $\sigma$-algebra $\mathcal{A}$ using $\mu$ is denoted by $E_{\mu}(f / \mathcal{A})$. We sometimes write $\mu(f)$ instead of $\int f d \mu$. If $\varphi \in C(X ; \mathbb{R})$, then an equilibrium state for $\varphi$ is some $\mu \in M(X, T)$ with $h_{\mu}(T)+\mu(\varphi)=P(T, \varphi)$ where $h_{\mu}(T)$ is the entropy of the measure-preserving transformation $T:(X, \mathcal{B}, \mu) \rightarrow(X, \mathcal{B}, \mu)$. An equivalent condition is $h_{\sigma}(T)+\sigma(\varphi) \leq$ $h_{\mu}(T)+\mu(\varphi) \forall \sigma \in M(X, T)$ ([W2]). The symbol $\rightarrow$ denotes uniform convergence.

If $n \geq 1$ and $\varphi: X \rightarrow \mathbb{R}$, we use $\left(T_{n} \varphi\right)(x)$ for $\sum_{i=0}^{n-1} \varphi\left(T^{i} x\right)$. Note that $\left(\mathcal{L}_{\varphi}^{n} f\right)(x)=$ $\sum_{y \in T^{-n} x} e^{\left(T_{n} \varphi\right)(y)} f(y)$.

For $\varphi \in C(X ; \mathbb{R}), n \geq 1, \delta>0$ define

$$
v_{n}(\varphi, \delta)=\sup \left\{\mid \varphi(x)-\varphi(z) \| d\left(T^{i} x, T^{i} z\right) \leq \delta, 0 \leq i \leq n-1\right\} .
$$

If $\delta$ is an expansive constant, then $v_{n}(\varphi, \delta) \rightarrow 0$ as $n \rightarrow \infty$.

The following result, in which $\mathcal{L}_{\varphi}^{*}$ denotes the dual of $\mathcal{L}_{\varphi}$, is well-known, and the first version of it was proved by Ruelle and is called the Ruelle operator theorem.

Theorem 1.1. Let $T: X \rightarrow X$ be a positively expansive local homeomorphism satisfying the weak specification condition. Let $\varphi \in C(X ; \mathbb{R})$ satisfy

$$
\sup _{n \geq 1} v_{n+j}\left(T_{n} \varphi, \delta\right) \rightarrow 0 \quad \text { as } j \rightarrow \infty \quad \text { for some } \delta>0
$$

(and hence for all smaller $\delta$ ). There exists $h \in C(X ; \mathbb{R})$ with $h>0, \lambda \in \mathbb{R}$ with $\lambda>0$, and $v \in M(X)$ so that $\mathcal{L}_{\varphi} h=\lambda h$ and $\mathcal{L}_{\varphi}^{*} \nu=\lambda \nu$. If we normalize $h$ so that $\nu(h)=1$, then $\forall f \in C(X ; \mathbb{R}) \frac{\left(\mathcal{L}_{\varphi}^{n} f\right)(x)}{\lambda^{n}} \rightrightarrows h(x) \nu(f)$. The spectral radius of $\mathcal{L}_{\varphi}: C(X ; \mathbb{R}) \rightarrow C(X ; \mathbb{R})$ is $\lambda$ and $\log \lambda=P(T, \varphi)$.

Proof. The condition on $\varphi$ still holds if we change to an equivalent metric. The result is a special case of Theorem 8 of [W1 because under a Reddy metric $D$ the map $T$ satisfies the conditions of [W1].

Since $v_{n+j}\left(T_{n} \varphi, \delta\right) \leq \sum_{i=j}^{j+n} v_{i}(\varphi, \delta)$, the condition on $\varphi$ is implied by $\sum_{n=1}^{\infty} v_{n}(\varphi, \delta)<\infty$

One consequence of Theorem 1.1 is that each $\varphi$ satisfying the condition of the theorem has a unique equilibrium state $\mu_{\varphi}$, given by $\mu_{\varphi}=h . \nu$ (i.e. $\mu_{\varphi}(f)=\nu(h . f)$ $\forall f \in C(X ; \mathbb{R}))$ and $T$ is exact with respect to $\mu_{\varphi}$. Also, under the conditions of the theorem one can show the natural extension of the measure-preserving transformation $T:\left(X, \mathcal{B}, \mu_{\varphi}\right) \rightarrow\left(X, \mathcal{B}, \mu_{\varphi}\right)$ is isomorphic to a Bernoulli shift ([W1]). 
We want to consider a weaker condition on $\varphi$ than the one in Theorem 1.1 It is worth pointing out that one needs no assumption on $\varphi \in C(X ; \mathbb{R})$ to get $\lambda>0$ and $\nu \in M(X)$ with $\mathcal{L}_{\varphi}^{*} \nu=\lambda \nu$, as is easily seen by using the Schauder-Tychanoff fixed point theorem on the map $\mu \rightarrow \frac{\mathcal{L}_{\varphi}^{*} \mu}{\left(\mathcal{L}_{\varphi}^{*} \mu\right)(1)}$ of $M(X)$. We shall consider the following condition that resembles one used by Bowen in the case of expansive homeomorphisms with a strong specification property ([B1]).

Definition. We say $\varphi \in C(X ; \mathbb{R})$ satisfies Bowen's condition (with respect to $T: X \rightarrow X)$ if $\exists \delta>0$ and $C>0$ with the property that whenever $d\left(T^{i} x, T^{i} z\right) \leq \delta$ for $0 \leq i \leq n-1$, then

$$
\left|\sum_{i=0}^{n-1}\left[\varphi\left(T^{i} x\right)-\varphi\left(T^{i} z\right)\right]\right| \leq C .
$$

Another way to phrase this definition is $\sup _{n \geq 1} v_{n}\left(T_{n} \varphi, \delta\right)<\infty$. If Bowen's condition holds for some $\delta$, then it holds for all smaller $\delta$. Notice that if $\varphi \in C(X ; \mathbb{R})$ satisfies the assumption of Theorem 1.1 then it satisfies Bowen's condition. There are examples of functions that satisfy Bowen's condition but not the assumption of Theorem 1.1. Later results will show the Bowen condition is a natural assumption (Theorem 4.8).

Bowen showed that when $T$ is an expansive homeomorphism with the strong specification property, then every continuous function satisfying his condition has a unique equilibrium state and this state is weak-mixing. He uses a method based on periodic points.

We consider how much of Ruelle's operator theorem holds for $\varphi \in C(X ; \mathbb{R})$ satisfying Bowen's condition. We prove there is a unique $\nu \in M(X)$ with $\mathcal{L}_{\varphi}^{*} \nu=\lambda \nu$ for $\lambda>0$ and this measure is tail-trivial. Recall that $\nu$ is tail-trivial means that the only values $\nu$ takes on sets in the tail $\sigma$-algebra $\mathcal{B}_{\infty}=\bigcap_{n=0}^{\infty} T^{-n} \mathcal{B}(X)$ are 0 and 1. Moreover, we show $\forall f \in C(X ; \mathbb{R}) \frac{\left(\mathcal{L}_{\varphi}^{n} f\right)(x)}{\left(\mathcal{L}_{\varphi}^{n} 1\right)(x)}$ converges uniformly to $\nu(f)$ (Theorem 2.16). We show $\varphi$ has a unique equilibrium state $\mu_{\varphi}$ and investigate some properties of $\mu_{\varphi}$. This is done by considering measurable $g$-functions after we construct a measurable $h: X \rightarrow\left[d_{1}, d_{2}\right] \subset(0, \infty)$ with $\mathcal{L}_{\varphi} h=\lambda h$ everywhere. We use a method suggested by the work of Fan who studied the case of continuous $g$-measures for subshifts of finite type ([F]). We also consider convergence of $\frac{\mathcal{L}_{\varphi}^{n} f}{\lambda^{n}}$ as $n \rightarrow \infty$ and obtain a result giving $L^{p}$ convergence. Such a result has been proved by Ruelle in a more general context in which the transformation $T$ need not be a local homeomorphism and hence the operator $\mathcal{L}_{\varphi}$ does not act on $C(X ; \mathbb{R})([\mathrm{Ru}])$. Our measurable $g$-function method allows us to deduce the $L^{p}$ convergence from a Martingale theorem.

In 42 we prove the convergence theorem (Theorem 2.16) and characterize the Bowen condition in terms of measures. In 3 we deduce results about $g$-measures from the results of $₫ 2$ We study equilibrium states and the convergence of $\frac{\mathcal{L}_{\varphi}^{n} f}{\lambda^{n}}$ in $\$ 4$ using measurable $g$-functions. In $\$ 5$ we consider continuity properties of a measurable density function obtained in 4 .

We now discuss the assumption that $T$ be a local homeomorphism. The condition can be stated in several equivalent ways. One statement, that is easier to check in examples, is that for every $x \in X$ there is an open neighbourhood $U_{x}$ of $x$ with $T U_{x}$ open such that $T$ maps $U_{x}$ homeomorphically onto $T U_{x}$. By a result of Eilenberg $(\underline{\mathrm{A}-\mathrm{H}}$, p. 31) this is equivalent to the following statement in which 
$\operatorname{diam}(B)$ denotes the diameter of the set $B \subset X$ : there exist $\delta_{1}>0, \theta>0$ and a function $\eta:\left(0, \delta_{1}\right] \rightarrow(0, \infty)$ with $\lim _{t \rightarrow 0} \eta(t)=0$ such that each open subset $V$ of $X$ with $\operatorname{diam}(V)<\delta_{1}$ has a decomposition of $T^{-1} V$ with the following properties:

(i) $T^{-1} V=U_{1} \cup \cdots \cup U_{k}$ for some $k$, where each $U_{i}$ is open;

(ii) $T$ maps each $U_{i}$ homeomorphically onto $V$;

(iii) if $i \neq j$, then $d\left(x_{i}, x_{j}\right) \geq \theta \forall x_{i} \in U_{i}, x_{j} \in U_{j}$;

(iv) $\operatorname{diam}(V)<\delta$ implies $\operatorname{diam}\left(U_{i}\right)<\eta(\delta)$ for each $i, 1 \leq i \leq k$, and each $\delta \in\left(0, \delta_{1}\right]$.

If we change to an equivalent metric, then this property still holds with different $\delta_{1}, \theta$ and $\eta$.

We now show that if $T: X \rightarrow X$ is a positively expansive local homeomorphism, there is some $\delta_{2}>0$ such that whenever $V$ is an open subset of $X$ with $\operatorname{diam}(V)<\delta_{2}$ and $x \in V$, then $\forall n \geq 1, T^{-n} V$ is a disjoint union $\bigcup_{y \in T^{-n} x} U_{y}$ of open sets with $T^{n}$ mapping each $U_{y}$ onto $V$. To see this choose a Reddy metric $D$ with constants $\tau>0, \lambda>1$, and let $\delta_{1}, \theta, \eta$ correspond to $D$ in the local homeomorphism condition. Let $\delta$ satisfy $\delta<\delta_{1}, \delta<\tau$ and $\eta(\delta)<\tau$ and suppose $V$ is open and $\operatorname{diam}_{D}(V)<\delta$ (where the subscript shows the diameter is taken for the metric $D$ ). Then $T^{-1} V=$ $U_{1} \cup \cdots \cup U_{k}$ where each open set $U_{i}$ has $\operatorname{diam}_{D}\left(U_{i}\right) \leq \delta / \lambda<\delta$. Therefore each $T^{-1} U_{i}$ can be decomposed into disjoint open sets of $D$-diameter at most $\delta / \lambda^{2}$. By induction we have $\forall n \geq 1 \forall x \in V T^{-n} V$ is a disjoint union $\bigcup_{y \in T^{-n}{ }_{x}} U_{y}$ of open sets of $D$-diameter at most $\delta / \lambda^{n}$ and $T^{n}$ maps each $U_{y}$ homeomorphically onto $V$ increasing distances. If we now revert to the original metric $d$ there is some $\delta_{2}>0$ such that if $V$ is open and $\operatorname{diam}(V)<\delta_{2}$ and $x \in v$, then $\forall n \geq 1 T^{-n} V$ is a disjoint union $\bigcup_{y \in T^{-n} x} U_{y}$ of open sets with $T^{n}$ mapping each $U_{y}$ homeomorphically onto $V$.

We now consider the condition of weak specification on $T$. Several equivalent forms are given in the following theorem in which $\widetilde{B}_{n}(x ; \varepsilon)$ denotes the closed Bowen ball $\left\{y \in X \mid d\left(T^{i} x, T^{i} y\right) \leq \varepsilon, 0 \leq i \leq n-1\right\}$.

Theorem 1.2. For a positively expansive local homeomorphism $T: X \rightarrow X$ the following statements are pairwise equivalent:

(i) $\forall \varepsilon>0 \exists N>0$ such that $\forall x \in X T^{-N} x$ is $\varepsilon$-dense in $X$.

(ii) $\forall \varepsilon>0 \exists M>0$ such that $\forall x, x^{\prime} \in X \forall n \geq 1 \exists w \in T^{-(n+M)} x^{\prime}$ with $d\left(T^{i} w, T^{i} x\right) \leq \varepsilon, 0 \leq i \leq n-1$.

(iii) $\forall \varepsilon>0 \exists M>0$ such that $\forall x \in X \forall n \geq 1 T^{n+M} \widetilde{B}_{n}(x ; \varepsilon)=X$.

(iv) $\forall \varepsilon>0 \exists M \geq 0$ such that $\forall x, x^{\prime} \in X \forall n_{1}, n_{2} \geq 1 \exists w \in X$ with $d\left(T^{i} w, T^{i} x\right)$ $\leq \varepsilon, 0 \leq i \leq n_{1}-1$ and $d\left(T^{n_{1}+M+j-1} w, T^{j} x_{2}\right) \leq \varepsilon, 0 \leq j \leq n_{2}-1$.

Proof. Note that each of the statements is independent of the metric. We use $B_{d}(x ; \varepsilon)$ for the open ball with center $x$ and radius $\varepsilon$ in the metric $d$.

Assume (i) holds and we prove (ii). Let $D$ be a Reddy metric with $D\left(T x, T x^{\prime}\right) \geq$ $\lambda D\left(x, x^{\prime}\right)$ whenever $D\left(x, x^{\prime}\right)<\tau$. Let $\delta_{1}, \theta, \eta$ be associated to $D$ by the local homeomorphism property. Let $\varepsilon>0$ satisfy $\varepsilon<\frac{\delta_{1}}{2}, \varepsilon<\tau / 2$ and $\eta(\varepsilon / 2)<\tau$, and let $N$ correspond to $\varepsilon$ in statement (i) for the metric $D$. By statement (i) choose $y_{n}^{\prime} \in T^{-N} x^{\prime}$ with $D\left(y_{n}^{\prime}, T^{n} x\right)<\varepsilon$. By the discussion before the statement of the theorem $T^{-n} B_{D}\left(T^{n} x ; \varepsilon\right)$ can be written as a disjoint union $\bigcup_{w \in T^{-n} T^{n} x} U_{w}$ of open sets with $D$-diameter at most $2 \varepsilon / \lambda^{n}$ and $T^{n}$ maps each $U_{w}$ homeomorphically onto 
$B_{D}\left(T^{n} x ; \varepsilon\right)$. Let $v_{n}^{\prime}=T^{-n} y_{n}^{\prime} \cap U_{x}$. Then $v_{n}^{\prime} \in T^{-(n+M)} x^{\prime}$ and for $0 \leq i \leq n-1$

$$
D\left(T^{i} x T^{i} v_{n}^{\prime}\right) \leq \frac{D\left(T^{n} x, T^{n} v_{n}^{\prime}\right)}{\lambda^{n-i}}<\varepsilon / \lambda^{n-i} .
$$
$d$.

Hence statement (ii) holds for the metric $D$, and therefore for the original metric

Statement (iii) is clearly the same as statement (ii), and it clearly implies statement (iv).

Now assume (iv) holds and we prove (i). Let $\varepsilon$ be an expansive constant and $x, z \in X$. We want to find $y \in T^{-N} x$ with $d(z, y)<\varepsilon$. By statement (iv) $\exists M$ so that $\forall n \geq 1 \exists w_{n} \in x$ with $d\left(w_{n}, z\right) \leq \varepsilon / 2$ and $d\left(T^{M+j} w_{n}, T^{j} x\right) \leq \varepsilon / 2$ for $0 \leq j \leq n-1$. Choose a convergent subsequence $w_{n_{i}} \rightarrow w$ to get $d(w, z) \leq \varepsilon / 2$ and $d\left(T^{M+j} w, T^{j} x\right) \leq \varepsilon / 2$ for all $j \geq 0$. Hence $T^{M} w=x$.

Because of statement (iv) we shall say that $T$ satisfies the weak specification condition if it satisfies one, and hence all, of the statements in Theorem 1.2. If $T$ satisfies the weak specification condition, then $T$ is topologically mixing.

Topologically mixing subshifts of finite type are examples of positively expansive local homeomorphisms with the weak specification property, and they are the only subshifts with these properties. Another important class of examples is given by expanding differentiable maps of smooth compact connected manifolds.

For details about $(n, \varepsilon)$ spanning sets and $(n, \varepsilon)$ separated sets see [W2].

Theorem 1.3. Let $T: X \rightarrow X$ be a positively expansive local homeomorphism satisfying the weak specification condition. Then $\forall \varphi \in C(X ; \mathbb{R}) \frac{1}{n} \log \left(\mathcal{L}_{\varphi}^{n} 1\right)(x) \rightarrow$ $P(T, \varphi)$.

Proof. By weak specification if $\varepsilon>0 \exists M$ so that $\forall x^{\prime} \in X \forall n \geq 1 T^{-(n+M)} x^{\prime}$ is $(n, \varepsilon)$ spanning, by statement (ii) of Theorem 1.2 If

$$
Q_{n}(\varphi, \varepsilon)=\inf \left\{\sum_{x \in F} e^{\left(T_{n} \varphi\right)(x)} \mid F \text { is }(n, \varepsilon) \text { spanning }\right\}
$$

then $\varepsilon$ is an expansive constant $P(T, \varphi)=\liminf \frac{1}{n} \log Q_{n}(\varphi, \varepsilon)$. So

$$
P(T, \varphi) \leq \liminf _{n \rightarrow \infty} \frac{1}{n}\left(\mathcal{L}_{\varphi}^{n+M} 1\right)\left(x^{\prime}\right)=\liminf _{n \rightarrow \infty} \frac{1}{n} \log \left(\mathcal{L}_{\varphi}^{n} 1\right)\left(x^{\prime}\right) \quad \forall x^{\prime} \in X .
$$

Since $T$ is positively expansive, $T^{-n} x^{\prime}$ is $(n, \varepsilon)$ separated if $\varepsilon$ is an expansive constant. If $P_{n}(\varphi, \varepsilon)=\sup \left\{\sum_{x \in E} e^{\left(T_{n} \varphi\right)(x)} \mid E\right.$ is $(n, \varepsilon)$ separated $\}$, then $P(T, \varphi) \geq$ $\limsup _{n \rightarrow \infty} \frac{1}{n} \log P_{n}(\varphi, \varepsilon)$, and this is actually an equality if $\varepsilon$ is an expansive constant. Therefore $P(T, \varphi) \geq \lim \sup _{n \rightarrow \infty} \frac{1}{n}\left(\mathcal{L}_{\varphi}^{n} 1\right)\left(x^{\prime}\right), \forall x^{\prime} \in X$. Hence

$$
\frac{1}{n} \log \left(\mathcal{L}_{\varphi}^{n} 1\right)(x) \rightrightarrows P(T, \varphi) .
$$

\section{Convergence theorem}

We adapt the method of Fan $([\mathrm{F}]$ ) to our more general situation. As before, let $X$ be a compact metric space with metric $d$ and let $T: X \rightarrow X$ be a positively expansive, local homeomorphism with the weak specification property. Let $\varphi \in C(X ; \mathbb{R})$ and let its transfer operator be $\mathcal{L}_{\varphi}: C(X ; \mathbb{R}) \rightarrow C(X ; \mathbb{R})$. Let 
$U_{T}: C(X ; \mathbb{R}) \rightarrow C(X ; \mathbb{R})$ be given by $\left(U_{T} f\right)(x)=f(T x)$. For $n \geq 1$ define $P_{\varphi}^{(n)}: C(X ; \mathbb{R}) \rightarrow C(X ; \mathbb{R})$ by

$$
\left(P_{\varphi}^{(n)} f\right)(x)=\frac{\left(U_{T}^{n} \mathcal{L}_{\varphi}^{n} f\right)(x)}{\left(U_{T}^{n} \mathcal{L}_{\varphi}^{n} 1\right)(x)}=\frac{\left(\mathcal{L}_{\varphi}^{n} f\right)\left(T^{n} x\right)}{\left(\mathcal{L}_{\varphi}^{n} 1\right)\left(T^{n} x\right)}=\frac{\sum_{z \in T^{-n} T^{n} x} e^{\left(T_{n} \varphi\right)(z)} f(z)}{\sum_{z \in T^{-n} T^{n} x} e^{\left(T_{n} \varphi\right)(z)}}
$$

These operators have the following properties.

Theorem 2.1. For $\varphi \in C(X ; \mathbb{R})$ and $n \geq 1$ let $P_{\varphi}^{(n)}$ be defined as above.

(i) $P_{\varphi}^{(n)}$ is linear, continuous and positive.

(ii) $P_{\varphi}^{(n)} 1=1$

(iii) If $\ell \in C(X ; \mathbb{R})$ is so that $\forall x \in X \ell$ is constant on $T^{-n} x$, then

$$
P_{\varphi}^{(n)}(\ell \cdot f)=\ell \cdot P_{\varphi}^{(n)} f \quad \forall f \in C(X ; \mathbb{R})
$$

(iv) $\mathcal{L}_{\varphi}^{n} P_{\varphi}^{(n)}=\mathcal{L}_{\varphi}^{n}$.

(v) If $n \leq m$, then $P_{\varphi}^{(n)} P_{\varphi}^{(m)}=P_{\varphi}^{(m)} P_{\varphi}^{(n)}=P_{\varphi}^{(m)}$.

(vi) $\forall f \in C(X ; \mathbb{R}) P_{\varphi}^{(n)} f$ is constant on each set $T^{-n} x$.

Proof. (i), (ii), (iii) and (vi) are clear from the definition. To prove (iv) we have

$$
\begin{aligned}
\left(\mathcal{L}_{\varphi}^{n} P_{\varphi}^{(n)} f\right)(x) & =\sum_{y \in T^{-n} x} e^{\left(T_{n} \varphi\right)(y)}\left(P_{\varphi}^{(n)} f\right)(y) \\
& =\sum_{y \in T^{-n} x} e^{\left(T_{n} \varphi\right)(y)} \frac{\sum_{z \in T^{-n} x} e^{\left(T_{n} \varphi\right)(z)} f(z)}{\sum_{z \in T^{-n} x} e^{\left(T_{n} \varphi\right)(z)}}=\left(\mathcal{L}_{\varphi}^{n} f\right)(x) .
\end{aligned}
$$

To prove (v) let $n \leq m$ and then

$$
\begin{gathered}
P_{\varphi}^{(n)} P_{\varphi}^{(m)} f=P_{\varphi}^{(m)} f \cdot P_{\varphi}^{(n)} 1 \quad \text { by (iii) and (vi) } \\
=P_{\varphi}^{(m)} f \quad \text { by (ii); } \\
P_{\varphi}^{(m)} P_{\varphi}^{(n)} f=\frac{U_{T}^{m} \mathcal{L}_{\varphi}^{m} P_{\varphi}^{(n)} f}{U_{T}^{m} \mathcal{L}_{\varphi}^{m} 1} \\
=\frac{U_{T}^{m} \mathcal{L}_{\varphi}^{m-n} \mathcal{L}_{\varphi}^{n} P_{\varphi}^{(n)} f}{U_{T}^{m} \mathcal{L}_{\varphi}^{m} 1}=\frac{U_{T}^{m} \mathcal{L}_{\varphi}^{m} f}{U_{T}^{m} \mathcal{L}_{\varphi}^{m} 1} \quad \text { by (iv) } \\
=P_{\varphi}^{(m)} f . \quad \square
\end{gathered}
$$

The dual operator $P_{\varphi}^{(n)^{*}}$ maps $M(X)$ into $M(X)$ and is continuous for the weak*topology. The set $K_{\varphi}^{(n)}=\left\{\nu \in M(X) \mid P_{\varphi}^{(n)^{*}} \nu=\nu\right\}$ is a compact convex set which is nonempty by the Schauder-Tychanoff fixed point theorem. Note that $K_{\varphi}^{(n)}=$ $P_{\varphi}^{(n)^{*}} M(X)$, by Theorem 2.1 (v). By Theorem 2.1 (v) we have $K_{\varphi}^{(1)} \supset K_{\varphi}^{(2)} \supset \cdots$ and $K_{\varphi}=\bigcap_{n=1}^{\infty} K_{\varphi}^{(n)}$ is a nonempty, compact convex set.

Let $\mathcal{L}_{\varphi}^{*}$ denote the dual of $\mathcal{L}_{\varphi}: C(X ; \mathbb{R}) \rightarrow C(X ; \mathbb{R})$.

Theorem 2.2. Let $J_{\varphi}: M(X) \rightarrow M(X)$ be defined by $J_{\varphi} \mu=\frac{\mathcal{L}_{\varphi}^{*} \mu}{\left(\mathcal{L}_{\varphi}^{*} \mu\right)(1)}$. Then $P_{\varphi}^{(n)^{*}} J_{\varphi}^{n}=J_{\varphi}^{n}$ and $J_{\varphi}^{n} M(X) \subset K_{\varphi}^{(n)}$

Proof. By induction $J_{\varphi}^{n} \mu=\frac{\left(\mathcal{L}_{\varphi}^{*}\right)^{n} \mu}{\left(\left(\mathcal{L}_{\varphi}^{*}\right)^{n} \mu\right)(1)}$. Then $P_{\varphi}^{(n)^{*}} J_{\varphi}^{n} \mu=J_{\varphi}^{n} \mu$ by Theorem 2 (iv).

Corollary 2.3. There exist $\nu \in M(X)$ and $\lambda>0$ with $\mathcal{L}_{\varphi}^{*} \nu=\lambda \nu$. Every such $\nu$ is in $K_{\varphi}$. 
Proof. The measures $\nu \in M(X)$ with $\mathcal{L}_{\varphi}^{*} \nu=\lambda \nu$ for some $\lambda>0$ are exactly the fixed points of $J_{\varphi}$. Since $J_{\varphi}$ has a fixed point by the Schauder-Tychanoff theorem, the result follows from Theorem 2.2

We shall use the following theorem to get information about $P_{\varphi}^{(n)^{*}}$ and about $K_{\varphi}^{(n)}$. If we let $L(X ; \mathbb{R})$ denote the vector space of all Borel measurable functions $f: X \rightarrow \mathbb{R}$, then we can consider $\mathcal{L}_{\varphi}$ as a $\operatorname{map} \mathcal{L}_{\varphi}: L(X ; \mathbb{R}) \rightarrow L(X ; \mathbb{R})$ and $P_{\varphi}^{(n)}$ as a map $P_{\varphi}^{(n)}: L(X ; \mathbb{R}) \rightarrow L(X ; \mathbb{R})$. We shall use the following result about an operator $P$ in the cases when $P$ is equal to $P_{\varphi}^{(n)}$ and later when $P$ is equal to $\mathcal{L}_{\varphi}$. For realvalued functions the expression $f_{n} \nearrow f$ means that for each $x, f_{1}(x) \leq f_{2}(x) \leq \cdots$ and $f_{n}(x) \rightarrow f(x)$.

Theorem 2.4. Let $P: L(X ; \mathbb{R}) \rightarrow L(X ; \mathbb{R})$ be a linear transformation which restricts to a continuous linear operator $P: C(X ; \mathbb{R}) \rightarrow C(X ; \mathbb{R})$. Assume $P$ is positive in the sense that if $f \in L(X ; \mathbb{R})$ and $f \geq 0$, then $P f \geq 0$. Let $P^{*}$ act on finite measures by $\int f d\left(P^{*} \mu\right)=\int P f d \mu, f \in C(X ; \mathbb{R})$. Assume that $P$ also has the property that whenever $\left\{f_{n}\right\}$ is a sequence in $L(X ; \mathbb{R})$ with $f_{n} \rightarrow f$ pointwise, then $P f_{n} \rightarrow P f$ pointwise. Let $\mu \in M(X)$. Then for all $f \in L(X ; \mathbb{R})$ with $f \geq 0$ we have $\int P f d \mu=\int f d\left(P^{*} \mu\right)$. In particular, $\forall B \in \mathcal{B}(X)\left(P^{*} \mu\right)(B)=\int P \chi_{B} d \mu$. Also, if $f \in L(X ; \mathbb{R})$, then $f \in L_{P^{*} \mu}^{1}(X)$ iff $P f \in L_{\mu}^{1}(X)$ and for such $f$ we have $\int P f d \mu=\int f d\left(P^{*} \mu\right)$.

Proof. Let $\mathcal{C}=\left\{B \in \mathcal{B}(X) \mid \int P \chi_{B} d \mu=\left(P^{*} \mu\right)(B)\right\}$. We have $X, \phi \in \mathcal{C}$ and $\mathcal{C}$ is closed under complements and finite disjoint unions. We show every open set is in $\mathcal{C}$. Let $U$ be an open subset of $X$ and write it as a countable union $\bigcup_{j=1}^{\infty} B\left(x_{j} ; r_{j}\right)$ of open balls. Let $C_{n}=\bigcup_{j=1}^{n} \overline{B\left(x_{j} ; r_{j}-\frac{1}{n}\right)}$ where $B(x ; r)$ is taken as empty if $r \leq 0$. Each $C_{n}$ is closed, $C_{1} \subset C_{2} \subset C_{3} \subset \cdots$ and $\bigcup_{n=1}^{\infty} C_{n}=U$. By Urysohn's lemma choose a continuous $f_{i}: X \rightarrow[0,1]$ with $\chi_{C_{i}} \leq f_{i} \leq \chi_{U}$. Then $f_{i} \rightarrow \chi_{U}$ pointwise so $P f_{i} \rightarrow P \chi_{U}$. By the dominated convergence theorem $\int P f_{i} d \mu \rightarrow \int P \chi_{U} d \mu$, and, also by the dominated convergence theorem for $P^{*} \mu, \int f_{i} d\left(P^{*} \mu\right) \rightarrow \int \chi_{U} d\left(P^{*} \mu\right)$. Hence $U \in \mathcal{C}$.

We next show $\mathcal{C}=\mathcal{B}(X)$. Define a measure $\mu_{1}$ on $X$ by $\mu_{1}(B)=\int P \chi_{B} d \mu$, $B \in \mathcal{B}(X)$. The above shows $\mu_{1}(U)=\left(P^{*} \mu\right)(U)$ for all open sets $U$, and since every finite measure on $X$ is regular, we have $\mu_{1}=P^{*} \mu$. Hence $\mathcal{C}=\mathcal{B}(X)$.

If $f$ is a nonnegative simple function, then $\int P f d \mu=\int f d P^{*} \mu$. If $f$ is a nonnegative measurable function, we can choose a sequence $\left\{f_{j}\right\}$ of nonnegative simple functions with $f_{j} \nearrow f$. Since $P$ is positive we have $P f_{j} \nearrow P f$ and the desired result follows for $f$, and $f \in L_{P^{*} \mu}^{1}(X)$ iff $P f \in L_{\mu}^{1}(X)$. By considering positive and negative parts of $f \in L(X ; \mathbb{R})$ we have $f \in L_{P^{*} \mu}^{1}(X)$ iff $P f \in L_{\mu}^{1}(X)$ and for such $f$ we have $\int P f d \mu=\int f d\left(P^{*} \mu\right)$.

The following result is now clear.

Corollary 2.5. If $\nu \in K_{\varphi}^{(n)}$ and $f \in L(X ; \mathbb{R})$, then $f \in L_{\nu}^{1}(X)$ iff $P_{\varphi}^{(n)} f \in L_{\nu}^{1}(X)$. For $f \in L_{\nu}^{1}(X)$ we have $\int f d \nu=\int P_{\varphi}^{(n)} f d \nu$.

Corollary 2.6. If $\nu \in K_{\varphi}^{(n)}, f \in L_{\nu}^{1}(X)$ and $B \in \mathcal{B}(X)$, then

$$
\int_{T^{-n} B} f d \nu=\int_{T^{-n} B} P_{\varphi}^{(n)} f d \nu .
$$


Proof. By Corollary 2.5 $\int f \chi_{T^{-n} B} d \nu=\int P_{\varphi}^{(n)}\left(f \cdot \chi_{T^{-n} B}\right) d \nu=\int \chi_{T^{-n} B} P_{\varphi}^{(n)} f d \nu$ by Theorem 2.1 (iii).

The following characterizes the members of $K_{\varphi}^{(n)}$.

Corollary 2.7. For $\nu \in M(X)$ the following statements are equivalent:

(i) $\nu \in K_{\varphi}^{(n)}$.

(ii) $E_{\nu}\left(f_{/ T^{-n} \mathcal{B}}\right)=P_{\varphi}^{(n)} f$ a.e. $(v) \forall f \in L_{\nu}^{1}(X)$.

(iii) $E_{\nu}\left(f_{/ T^{-n} \mathcal{B}}\right)=P_{\varphi}^{(n)} f$ a.e. $(v) \forall f \in C(X ; \mathbb{R})$.

Proof. We have (i) $\Rightarrow$ (ii) by Corollary [2.6] Clearly (ii) $\Rightarrow$ (iii). If (iii) holds and $f \in C(X ; \mathbb{R})$, then

$$
\int P^{(n)} f d \nu=\int E_{\nu}\left(f_{/ T^{-n} \mathcal{B}}\right) d \nu=\int f d \nu, \text { so } \nu \in K_{\varphi}^{(n)} .
$$

So the elements of $K_{\varphi}^{(n)}$ are those probability measures with conditional expectation $E\left(f / T^{-n} \mathcal{B}\right)$ given by $P_{\varphi}^{(n)} f$.

Corollary 2.8. Let $\nu \in M(X)$ satisfy $\mathcal{L}_{\varphi}^{*} \nu=\lambda \nu$ for $\lambda \geq 0$. If $f \in L(X ; \mathbb{R})$ and $f \geq 0$ we have $\int \mathcal{L}_{\varphi} f d \mu=\lambda \int f d \nu$. Also, for $f \in L(X ; \mathbb{R})$ we have $f \in L_{\nu}^{1}(X)$ iff $\mathcal{L}_{\varphi} f \in L_{\nu}^{1}(X)$ and for these $f, \int \mathcal{L}_{\varphi} f d \nu=\lambda \int f d \nu$.

Proof. Put $P$ equal to $\mathcal{L}_{\varphi}$ in Theorem [2.4.

The following result characterizes the situation when $K_{\varphi}$ is as small as possible.

Theorem 2.9. Let $T: X \rightarrow X$ be a positively expansive local homeomorphism with the weak specification property and let $\varphi \in C(X ; \mathbb{R})$. The following statements are pairwise equivalent:

(i) $K_{\varphi}$ has only one member.

(ii) $\forall f \in C(X ; \mathbb{R}) \exists c(f) \in \mathbb{R}$ with $P_{\varphi}^{(n)} f \rightrightarrows c(f)$.

(iii) $\forall f \in C(X ; \mathbb{R}) \exists c(f) \in \mathbb{R}$ with $P_{\varphi}^{(n)} f \rightarrow c(f)$ pointwise.

(iv) $\forall f \in C(x ; \mathbb{R}) \exists c(f) \in \mathbb{R}$ with $\frac{\mathcal{L}_{\varphi}^{n} f}{\mathcal{L}_{\varphi}^{n} 1} \rightarrow c(f)$.

When these statements hold the unique element $\nu$ of $K_{\varphi}$ satisfies $\mathcal{L}_{\varphi}^{*} \nu=\lambda \nu$ for some $\lambda>0$ and $c(f)=\int f d \nu$.

Proof. (i) $\Rightarrow$ (ii). Let $K_{\varphi}=\{\nu\}$. If (ii) holds, then $c(f)=\int f d \nu$ by the bounded convergence theorem. If (ii) fails, then $\exists f_{0} \in C(X ; \mathbb{R})$ and $\exists \varepsilon_{0}>0$ and sequences $n_{j} \rightarrow \infty$ and $x_{j} \in X$ with $\left|\left(P_{\varphi}^{\left(n_{j}\right)} f_{0}\right)\left(x_{j}\right)-\nu\left(f_{0}\right)\right| \geq \varepsilon_{0} \forall j \geq 1$. We can write this

$$
\left|\int f_{0} d\left(P_{\varphi}^{\left(n_{j}\right)^{*}} \delta_{x_{j}}\right)-\int f_{0} d \nu\right| \geq \varepsilon_{0} \quad \forall \geq 1 .
$$

Choose a convergent subsequence $P_{\varphi}^{\left(n_{j}\right)^{*}} \delta_{x_{j}} \rightarrow \tau \in M(X)$. Since $K_{\varphi}^{(n)}=$ $P_{\varphi}^{(n)^{*}} M(X)$, we have $\tau \in K_{\varphi}$. But $\left|\int f_{0} d \tau-\int f_{0} d \nu\right| \geq \varepsilon_{0}$ so $\tau \neq \nu$ and this contradicts (i).

It is clear that (ii) and (iv) are equivalent, and that (ii) implies (iii). It remains to show (ii) implies (i). If (iii) holds and $\nu \in K_{\varphi}$, the bounded convergence theorem gives $\int f d \nu=c(f) \forall f \in C(X ; \mathbb{R})$ so that $\nu$ is uniquely determined. 
The above proof gives the following more general theorem. Let $X$ be a compact metric space and for each $n \geq 1$ let $P_{n}: C(X ; \mathbb{R}) \rightarrow C(X ; \mathbb{R})$ be linear, continuous, positive operators with $P_{n}(1)=1$ and $P_{n} P_{m}=P_{\max (n, m)}$. Define $K_{n}=\{\nu \in$ $\left.M(X) \mid P_{n}^{*} \nu=\nu\right\}$ and then $K_{1} \supset K_{2} \supset \cdots$ and $K_{\infty}=\bigcap_{1}^{\infty} K_{n}$ is nonempty. One can show $K_{\infty}$ has only one member iff $\forall f \in C(X ; \mathbb{R}) \exists c(f) \in \mathbb{R}$ with $P_{n} f \Rightarrow c(f)$.

We want to investigate the extreme points of $K_{\varphi}$, and the following lemma is helpful.

Lemma 2.10. Let $\nu \in K_{\varphi}^{(n)}$ and let $h: X \rightarrow[0, \infty)$ be measurable and $\int h d \nu=1$. Then $h \cdot \nu \in K_{\varphi}^{(n)}$ iff $P_{\varphi}^{(n)} h=h$ a.e. $(v)$.

Proof. If $h \cdot \nu \in K_{\varphi}^{(n)}$, then $\forall A \in \mathcal{B}(X)$

$$
\begin{aligned}
\int_{A} h d \nu=\int \chi_{A} \cdot h d \nu & =\int\left(P^{(n)} \chi_{A}\right) \cdot h d \nu \text { by Corollary 2.5 applied to } h \cdot \nu \\
& =\int P^{(n)}\left(P^{(n)} \chi_{A} \cdot h\right) d \nu \text { by Corollary 2.5 applied to } \nu \\
& =\int P^{(n)} \chi_{A} \cdot P^{(n)} h d \nu \text { by Theorem 2.1 (iii) \& (vi) } \\
& =\int P^{(n)}\left(\chi_{A} \cdot P^{(n)} h\right) d \nu \text { by Theorem 2.1 (iii) \& (vi) } \\
& =\int \chi_{A} \cdot P^{(n)} h d \nu \text { by Corollary 2.5 applied to } \nu \\
& =\int P_{A}^{(n)} h d \nu .
\end{aligned}
$$

Hence $P^{(n)} h=h$ a.e. $(v)$.

Conversely, if $P^{(n)} h=h$ a.e. $(v)$, then for $f \in C(X ; \mathbb{R})$,

$$
\begin{aligned}
\int f \cdot h d \nu & =\int P^{(n)}(f \cdot h) d \nu \text { by Corollary 2.5 applied to } \nu \\
& =\int P^{(n)} f \cdot h d \nu \text { by Theorem 2.1 (iii). }
\end{aligned}
$$

Therefore $h \cdot \nu \in K^{(n)}$.

This leads to

Theorem 2.11. (i) The extreme points of $K_{\varphi}$ are exactly the elements of $K_{\varphi}$ that are tail-trivial.

(ii) If $\nu_{1}, \nu_{2}$ are extreme points of $K_{\varphi}$, then either $\nu_{1}=\nu_{2}$ or $\nu_{1}, \nu_{2}$ are singular on $\mathcal{B}_{\infty}=\bigcap_{n=0}^{\infty} T^{-n} \mathcal{B}(X)$.

Proof. (i) Let $\nu$ be tail-trivial and $\nu \in K_{\varphi}$. Let $\nu=p \nu_{1}+(1-p) \nu_{2}$ with $0<p<1$ and $\nu_{1}, \nu_{2} \in K_{\varphi}$. Since $\nu_{1} \ll \nu$ and $\nu_{2} \ll \nu$, the above lemma gives $\nu_{1}=h_{1} \nu$, $\nu_{2}=h_{2} \nu$ with $h_{1}, h_{2}$ both measurable $(\bmod \nu)$ with respect to $\mathcal{B}_{\infty}$. Therefore $h_{1}, h_{2}$ are both constant $(\bmod \nu)$ so $\nu_{1}=\nu=\nu_{2}$. Hence $\nu$ is an extreme point of $K_{\varphi}$

If $\nu \in K_{\varphi}$ and $\nu$ is not tail-trivial, there is some $B_{0} \in \mathcal{B}_{\infty}$ with $0<\nu\left(B_{0}\right)<1$. Then $\frac{\chi_{B_{0}} \cdot \nu}{\nu\left(B_{0}\right)}, \frac{\chi_{X \backslash B_{0}} \cdot \nu}{\nu\left(X \backslash B_{0}\right)} \in K_{\varphi}$ by Lemma 2.10 and $\nu=\nu\left(B_{0}\right) \cdot\left(\frac{\chi_{B_{0}} \cdot \nu}{\nu\left(B_{0}\right)}\right)+$ $\left(1-\nu\left(B_{0}\right)\right)\left(\frac{\chi_{X \backslash B_{0}} \cdot \nu}{\nu\left(X \backslash B_{0}\right)}\right)$ so that $\nu$ is not an extreme point of $K_{\varphi}$. 
(ii) Let $\nu_{1}, \nu_{2} \in K_{\varphi}$ and both be tail-trivial. If $\nu_{1} \neq \nu_{2}$, choose $f_{0} \in C(X ; \mathbb{R})$ $\nu_{1}\left(f_{0}\right) \neq \nu_{2}\left(f_{0}\right)$. Let $B_{i}=\left\{x \in X \mid\left(P_{n} f_{0}\right)(x) \rightarrow \nu_{i}\left(f_{0}\right)\right\}$. By the Martingale theorem $\nu_{i}\left(B_{i}\right)=1\left([\mathrm{P}]\right.$, p. 231). Since $B_{1} \cap B_{2}=\varnothing$ we have that $\nu_{1}, \nu_{2}$ are a singular pair. Note that $B_{i} \in \mathcal{B}_{\infty}$.

We want to show that when $\varphi$ satisfies Bowen's condition there are no singular pairs in $\varphi$, and hence, by Theorem 2.11 (ii), $K_{\varphi}$ as only one member. We shall use the open Bowen balls $B_{n}(x ; \delta)=\left\{y \in X: \mid d\left(T^{i} x, T^{i} y\right)<\delta, 0 \leq i \leq n-1\right\}$. For $M \geq 1$ and $\varepsilon>0$ let $s_{M}(\varepsilon)$ denote the maximum number of points in a $(M, \varepsilon)$ separated set with respect to $T$ ([W2], p. 169). For the following lemma we only need $T$ to be positively expansive.

Lemma 2.12. If $T: X \rightarrow X$ is positively expansive and $2 \delta$ is an expansive constant, then for $M \geq 1, n \geq 0$ and $y, z \in X$ the set $B_{n}(y ; \delta) \cap T^{-(n+M)} z$ has at most $s_{M}(2 \delta)$ points.

Proof. If $w_{1}, w_{2} \in B_{n}(y ; \delta) \cap T^{-(n+M)} z$, then $d\left(T^{i} w_{1}, T^{i} w_{2}\right)<2 \delta$ for $0 \leq i \leq n-1$ and $T^{n+M} w_{1}=T^{n+M} w_{2}$. Since $2 \delta$ is an expansive constant, the set $T^{n}\left(B_{n}(y ; \delta) \cap\right.$ $\left.T^{-(n+M)} z\right)$ has the same cardinality as $B_{n}(y ; \delta) \cap T^{-(n+m)} z$, and it must be $(M, 2 \delta)$ separated.

Corollary 2.13. With the notation of Lemma 2.12 $\left\{v \in T^{-(n+M)} z \mid B_{n}\left(v ; \delta_{/ 2}\right) \cap\right.$ $\left.B_{n}\left(y ; \delta_{/ 2}\right) \neq \varnothing\right\}$ has at most $s_{M}(2 \delta)$ points.

Proof. Let $v$ be in the above set. Then $v \in B_{n}(y ; \delta)$ so $v \in T^{-(n+m)} z \cap B_{n}(y ; \delta)$, and we can apply Lemma 2.12

The following theorem concerns strictly positive continuous functions on $X$, and we can write such a function as $f=e^{F}$ for $F \in C(X ; \mathbb{R})$.

Theorem 2.14. Let $T: X \rightarrow X$ be a positively expansive local homeomorphism satisfying the weak specification property. Let $\varphi \in C(X ; \mathbb{R})$. For sufficiently small $\delta>0 \exists M \geq 1$ with the property that $\forall n \geq 1, \forall x, z \in X, \forall F \in C(X ; \mathbb{R})$

$$
\left(\mathcal{L}_{\varphi}^{n+M} e^{F}\right)(x) \leq e^{v_{n}(F, \delta)} A_{n}\left(\mathcal{L}_{\varphi}^{n+M} e^{F}\right)(z)
$$

where $A_{n}=s_{M}(2 \delta) e^{M\|\varphi\|+v_{n}\left(T_{n} \varphi, \delta\right)}$.

Proof. Let $\delta>0$ be so small that $2 \delta$ is an expansive constant. By weak specification there exist $M \geq 1$ with $T^{n+M} \widetilde{B}_{n}(w ; \delta / 4)=X \forall n \geq 1, \forall w \in X$. Hence $\forall z, y \in$ $X \forall n \geq 1 B_{n}\left(y ; \delta_{/ 2}\right) \cap T^{-(n+M)} z \neq \varnothing$.

Let $F \in C(X ; \mathbb{R})$ and $z, x \in X$. Then $\sum_{y \in T^{-(n+M)} x} e^{\left(T_{n+M} \varphi\right)(y)} e^{F(y)} \leq$ $e_{M}(2 \delta) \sum_{\nu \in T^{-(n+M)} z} e^{\left(T_{n+M} \varphi\right)(z)+M\|\varphi\|+v_{n}\left(T_{n} \varphi, \delta\right)} e^{F(v)+v_{n}(F, \delta)}$ by associating to each $y \in T^{-(n+M)} x$ those $v \in T^{-(n+M)} z$ with $B_{n}\left(y ; \delta_{/ 2}\right) \cap B_{n}(v ; \delta / 2) \neq \varnothing$. For each $y$ this set of $v$ 's is nonempty by choice of $M$ above, and has at most $s_{M}(2 \delta)$ members by Corollary 2.13,

This gives $\left(\mathcal{L}_{\varphi}^{n+M} e^{F}\right)(x) \leq e^{v_{n}(F, \delta)} A_{n}\left(\mathcal{L}_{\varphi}^{n+M} e^{F}\right)(z)$.

Corollary 2.15. Let $T, \varphi$ be as in Theorem 2.14, and for sufficiently small $\delta>0$ let $M$ be given by Theorem 2.14. Then $\forall n \geq 1, \forall x, z \in X, \forall F \in C(X ; \mathbb{R})$

$$
\left(P_{\varphi}^{n+M} e^{F}\right)(x) \leq e^{v_{n}(F, \delta)} A_{n}^{2}\left(P_{\varphi}^{n+M} e^{F}\right)(z),
$$

where $A_{n}=s_{M}(2 \delta) e^{M\|\varphi\|+v_{n}\left(T_{n} \varphi, \delta\right)}$. 
Proof. By Theorem 2.14 we have

$$
\left(\mathcal{L}_{\varphi}^{n+M} e^{F}\right)\left(T^{n+M} x\right) \leq e^{v_{n}(F, \delta)} A_{n}\left(\mathcal{L}_{\varphi}^{n+M} e^{F}\right)\left(T^{n+M} z\right),
$$

and by Theorem 2.14 with $F=0$ we have

$$
\left(\mathcal{L}_{\varphi}^{n+M} 1\right)\left(T^{n+M} z\right) \leq A_{n}\left(\mathcal{L}_{\varphi}^{n+M} 1\right)\left(T^{n+M} x\right) .
$$

Theorem 2.16. Let $T: X \rightarrow X$ be a positively expansive local homeomorphism satisfying the weak specification condition. Let $\varphi \in C(X ; \mathbb{R})$ satisfy $\liminf _{n \rightarrow \infty} v_{n}\left(T_{n} \varphi, \delta\right)<\infty$ for some $\delta>0$, which is implied by Bowen's condition. Then:

(i) $K_{\varphi}$ has only one member which is the unique $\nu \in M(X)$ with $\mathcal{L}_{\varphi}^{*} \nu=\lambda \nu$ for some $\lambda>0$.

(ii) $\nu$ is tail-trivial.

(iii) $\forall f \in C(X ; \mathbb{R})\left(\frac{\mathcal{L}_{\varphi}^{n} f}{\mathcal{L}_{\varphi}^{n} 1}\right)(x) \rightrightarrows \nu(f)$.

(iv) The unique $\lambda>0$ determined by (i) satisfies

$$
\lambda=\int \mathcal{L}_{\varphi} 1 d \nu=e^{P(T, \varphi)}=\lim _{n \rightarrow \infty}\left(\frac{\mathcal{L}_{\varphi}^{n+1} 1}{\mathcal{L}_{\varphi}^{n} 1}\right)(x) \forall x \in X .
$$

Also, $\lambda$ is the spectral radius of $\mathcal{L}_{\varphi}: C(X ; \mathbb{R}) \rightarrow C(X ; \mathbb{R})$.

(v) If $\varphi$ satisfies Bowen's condition, there is a constant $D>1$ so that $D^{-1} \leq$ $\frac{\mathcal{L}_{\varphi}^{n} 1(x)}{\lambda^{n}} \leq D \forall n \geq 0, \forall x \in X$.

Proof. We first use Corollary2.15 to show $K_{\varphi}$ has only one member. let $\nu, \nu^{\prime} \in K_{\varphi}$. If we integrate the inequality in Corollary 2.15 in $x$ with respect to $\nu$, and integrate in $z$ with respect to $\nu^{\prime}$ we get

$$
\int e^{F} d \nu \leq e^{v_{n}(F, \delta)} A_{n}^{2} \int e^{F} d \nu^{\prime} \quad \forall n \geq 1, \forall F \in C(X ; \mathbb{R}) .
$$

If $A=\liminf _{n \rightarrow \infty} A_{n}$, which is finite by assumption, then

$$
\int e^{F} d \nu \leq A^{2} \int e^{F} d \nu^{\prime} \quad \forall f \in C(X ; \mathbb{R})
$$

This implies $\nu \ll \nu^{\prime}$, so by Theorem 2.11 (ii) we have $\nu=\nu^{\prime}$. To see the above inequality gives $\nu \ll \nu^{\prime}$, let $C$ be a closed subset of $X$ with $\nu^{\prime}(C)=0$. For $\varepsilon>0$ choose an open set $U_{\varepsilon} \supset C$ with $\nu^{\prime}\left(U_{\varepsilon}\right)<\varepsilon$, and a Urysohn function $f \in C(X ; \mathbb{R})$ with $\chi_{C} \leq f \leq \chi_{U_{\varepsilon}}$. Then $\forall \tau>0 \int(f+\tau) d \nu \leq A^{2} \int(f+\tau) d \nu^{\prime}$ so $\nu(C)+\tau \leq A^{2}\left(\nu^{\prime}\left(U_{\varepsilon}\right)+\tau\right) \leq A^{2}(\varepsilon+\tau)$, and hence $\nu(C)=0$. If $B \in \mathcal{B}(X)$ has $\nu^{\prime}(B)=0$, then $\nu^{\prime}(C)=0$ for every closed $C \subset B$ and so $\nu(B)=\sup \{\nu(C) \mid C$ closed, $C \subset B\}=0$. Hence $K_{\varphi}$ has only one member.

From Corollary 2.3 we know there exists $\nu \in M(X)$ and $\lambda>0$ with $\mathcal{L}_{\varphi}^{*} \nu=\lambda \nu$, and that such a measure is in $K_{\varphi}$. Hence there is a unique such $\nu$. Then $\lambda$ is given by $\lambda=\int \mathcal{L}_{\varphi} 1 d \nu$. Theorem 2.11 (i) gives that $\nu$ is tail-trivial.

The convergence property (iii) follows by Theorem 2.9. If we put $f=\mathcal{L}_{\varphi} 1$ in the convergence property we get $\frac{\mathcal{L}_{\varphi}^{n+1} 1}{\mathcal{L}_{\varphi}^{n} 1}(x) \Rightarrow \lambda$. Hence $\left[\left(\mathcal{L}_{\varphi}^{n} 1\right)(x)\right]^{1 / n} \Rightarrow \lambda$ and since $\left\|\mathcal{L}_{\varphi}^{n}\right\|=\left\|\mathcal{L}_{\varphi}^{n} 1\right\|_{\infty}$, we get that $\lambda$ is the spectra radius of $\mathcal{L}_{\varphi}: C(X ; \mathbb{R}) \rightarrow C(X ; \mathbb{R})$.

The relation $P(T, \varphi)=\log \lambda$ follows from $\frac{1}{n} \mathcal{L}_{\varphi}^{n} 1(x) \rightarrow \log \lambda$ and Theorem 1.2

To prove (v) let $\delta$ be small enough for Theorem 2.14 to hold and let $M$ be given by Theorem 2.14. Putting $F \equiv 0$ in Theorem 2.14 gives

$$
\mathcal{L}_{\varphi}^{n+M} 1(x) \leq A_{\infty} \mathcal{L}_{\varphi}^{n+M} 1(z) \quad \forall n \geq 1, \quad \forall x, z \in X
$$


where $A_{\infty}=\sup A_{n}$. If we integrate with $\nu$ in $z$ we get $\frac{\mathcal{L}_{\varphi}^{n+M} 1(x)}{\lambda^{n+M}} \leq A_{\infty} \forall x \in$ $X, \forall n \geq 1$, and if we integrate in $x$ we get $A_{\infty}^{-1} \leq \frac{\mathcal{L}_{\varphi}^{n+M} 1(z)}{\lambda^{n+M}} \forall z \in X, \forall n \geq 1$. If we let $B=\sup \left\{\frac{\mathcal{L}_{\varphi}^{i} 1(x)}{\lambda^{i}} \mid 0 \leq i \leq M, x \in X\right\}$ and $E=\inf \left\{\frac{\mathcal{L}_{\varphi}^{i} 1(x)}{\lambda^{i}} \mid 0 \leq i \leq M, x \in X\right\}>$ 0 , then put $D=\max \left(A_{\infty}, B, E^{-1}\right)$. Then $D^{-1} \leq \frac{\mathcal{L}_{\varphi}^{n} 1(x)}{\lambda^{n}} \leq D \forall x \in X, \forall n \geq 0$.

Corollary 2.17. Let $T$ be a positively expansive local homeomorphism satisfying the weak specification condition, and let $\varphi \in C(X ; \mathbb{R})$ satisfy Bowen's condition. If $\nu, \lambda$ are as in Theorem 2.16 the following statements are pairwise equivalent:

(i) $\exists h \in C(X ; \mathbb{R})$ with $h>0, \mathcal{L}_{\varphi} h=\lambda h, \nu(h)=1$ and

$$
\forall f \in C(X ; \mathbb{R}) \frac{\left(\mathcal{L}_{\varphi}^{n} f\right)(x)}{\lambda^{n}} \rightrightarrows h(x) \nu(f) .
$$

(ii) $\exists h \in C(X ; \mathbb{R})$ with $h>0$ and $\mathcal{L}_{\varphi} h=\lambda h$.

(iii) $\frac{\left(\mathcal{L}_{\varphi}^{n} 1\right)(x)}{\lambda^{n}}$ converges uniformly as $n \rightarrow \infty$.

(iv) $\frac{1}{n} \sum_{i=0}^{n-1} \frac{\left(\mathcal{L}_{\varphi}^{i} 1\right)(x)}{\lambda^{i}}$ converges uniformly as $n \rightarrow \infty$.

(v) $\left\{\frac{\mathcal{L}_{\varphi}^{n} 1}{\lambda^{n}} \mid n \geq 0\right\}$ is an equicontinuous subset of $C(X ; \mathbb{R})$.

(vi) $\left\{\frac{1}{n} \sum_{i=0}^{n-1} \frac{\mathcal{L}_{\varphi}^{i} 1}{\lambda^{i}} \mid n \geq 1\right\}$ is an equicontinuous subset of $C(X ; \mathbb{R})$.

Proof. Clearly (i) $\Rightarrow$ (ii). If (ii) holds, then putting $f=h$ in Theorem 2.16 (iii) gives $\frac{\left(\mathcal{L}_{\varphi}^{n} 1\right)(x)}{\lambda^{n}} \rightrightarrows \frac{h(x)}{\nu(h)}$, so (iii) holds. Clearly (iii) $\Rightarrow$ (iv), (iv) $\Rightarrow($ vi), (iii) $\Rightarrow$ (v) and $(\mathrm{v}) \Rightarrow(\mathrm{vi})$. It remains to show (vi) $\Rightarrow$ (i). If (vi) holds, then $\left\{\frac{1}{n} \sum_{i=0}^{n-1} \frac{\mathcal{L}_{\varphi}^{i} 1}{\lambda^{i}} \mid n \geq 1\right\}$ is equicontinuous and each member is bounded from below by $D^{-1}$ and above by $D$, by Theorem 2.16 (v). It's closure in $C(X ; \mathbb{R})$ is therefore compact so that there is a subsequence $\frac{1}{n_{j}} \sum_{i=0}^{n_{j}-1} \frac{\mathcal{L}_{\varphi}^{i} 1}{\lambda^{i}}$ which converges in $C(X ; \mathbb{R})$ to some $h$ with $D^{-1} \leq h$. Then $\mathcal{L}_{\varphi} h=\lambda h$, and $\nu(h)=1$. Putting $f=h$ in Theorem 2.16 (iii) gives $\frac{\left(\mathcal{L}_{\varphi}^{n} 1\right)(x)}{\lambda^{n}} \rightarrow h(x)$ and hence Theorem 2.16 (iii) gives $\frac{\mathcal{L}_{\varphi}^{n} f}{\lambda^{n}}=h \nu(f)$.

Due to Theorem 2.16 (v), part (v) of Corollary2.17] is equivalent to $\forall \varepsilon>0 \exists \delta>0$ such that whenever $d\left(x, x^{\prime}\right)<\delta$, then $\left|\frac{\left(\mathcal{L}_{\varphi}^{n} 1\right)(x)}{\left(\mathcal{L}_{\varphi}^{n} 1\right)\left(x^{\prime}\right)}-1\right|<\varepsilon \forall n \geq 1$.

We shall deduce uniqueness of the equilibrium state of a Bowen function in 4 and also consider convergence of $\frac{\mathcal{L}_{\varphi}^{n} f}{\lambda^{n}}$.

One has the following characterization of the Bowen condition.

Theorem 2.18. Let $T: X \rightarrow X$ be a positively expansive local homeomorphism satisfying the weak specification property. For $\varphi \in C(X ; \mathbb{R})$ the following are pairwise equivalent:

(i) $\varphi$ satisfies Bowen's condition.

(ii) $\exists \nu \in M(X)$ and $\lambda>0$ with the property that for all sufficiently small $\delta>$ $0 \exists D_{\delta}>1$ with $D_{\delta}^{-1} \leq \frac{\nu\left(B_{n}(x ; \delta)\right)}{\lambda^{-n} e^{\left(T_{n} \varphi\right)(x)}} \leq D_{\delta} \forall x \in X, \forall n \geq 1$.

(iii) $\exists \nu \in M(X)$ and $\lambda>0$ with the property that for some expansive constant

$$
\delta_{0} \exists D_{\delta_{0}}>1 \text { with } D_{\delta_{0}}^{-1} \leq \frac{\nu\left(B_{n}\left(x ; \delta_{0}\right)\right)}{\lambda^{-n} e^{\left(T_{n} \varphi\right)(x)}} \leq D_{\delta_{0}} \quad \forall x \in X, \quad \forall n \geq 1 .
$$

Proof. To show that (ii) and (iii) are equivalent it suffices to show that if $\delta_{1}, \delta_{2}$ are expansive constants and the property holds for $\delta_{1}$, then it holds for $\delta_{2}$. So assume $D_{\delta_{1}}$ exists. Choose $N$ so that $d\left(T^{i} x, T^{i} y\right)<\delta_{1}, 0 \leq i \leq N-1$, implies $d(x, y)<\delta_{2}$ 
and choose $K$ so that $d\left(T^{i} x, T^{i} y\right)<\delta_{2}, 0 \leq i \leq K-1$, implies $d(x, y)<\delta_{1}$. Then $B_{n+N}\left(x ; \delta_{1}\right) \subset B_{n}\left(x ; \delta_{2}\right)$ so

$$
\left(\frac{1}{\lambda e^{\|e\|}}\right)^{N} D_{\delta_{2}}^{-1} \leq \frac{\nu\left(B_{n}\left(x ; \delta_{2}\right)\right)}{\lambda^{-n} e^{\left(T_{n} \varphi\right)(x)}} \quad \forall x \in X, \quad \forall n \geq 1,
$$

and $B_{n}\left(x ; \delta_{2}\right) \subset B_{n-K}\left(x ; \delta_{1}\right)$ for $n \geq K$ so $\frac{\nu\left(B_{n}\left(x ; \delta_{2}\right)\right)}{\lambda-n_{e}\left(T_{n} \varphi\right)(x)} \leq D_{\delta_{2}}\left(\lambda e^{\|e\|}\right)^{K}$ for $n \geq K$. If $C=\sup \left\{\frac{\nu\left(B_{n}\left(x ; \delta_{2}\right)\right)}{\lambda^{-n} e^{\left(T_{n} \varphi\right)(x)}} \mid x \in X, 1 \leq n \leq K\right\}$, then put

$$
D_{\delta_{2}}=\max \left(C, D_{\delta_{1}}\left(\lambda e^{\|e\|}\right)^{\max (K, N)}\right)
$$

Hence (ii) and (iii) are equivalent.

Assume (i) holds and let $\delta>0$ be so small that it is an expansive constant and $C=\sup _{n \geq 1} v_{n}\left(T_{n} \varphi, \delta\right)<\infty$. Let $\nu \in M(X)$ and $\lambda>0$ be so that $\mathcal{L}^{*} \nu=\lambda \nu$. By Corollary $2.8 \int \mathcal{L}_{\varphi} f d \nu=\lambda \int f d \nu$ for $f \in L(X ; \mathbb{R})$ with $f \geq 0$. By weak specification there is $M \geq 1$ with $T^{n+M} \widetilde{B}_{n}(x ; \delta)=X \forall x \in X, \forall n \geq 1$. Then

$$
\mathcal{L}_{\varphi}^{n+M} \chi_{B_{n}(x ; \delta)}(z)=\sum_{y \in T^{-(n+M)} z} e^{\left(T_{n+M} \varphi\right)(y)} \chi_{B_{n}(x ; \delta)}(y)
$$

has nonzero terms for each $z \in X$ and $e^{\left(T_{n} \varphi\right)(x)-M\|\varphi\|-C} \leq \mathcal{L}_{\varphi}^{n+M} \chi_{B_{n}(x ; \delta)}(z) \leq$ $s_{M}(2 \delta) e^{T_{n} \varphi(x)+M\|\varphi\|+C}$ by Lemma 2.12, Integrating in $z$ gives $e^{\left(T_{n} \varphi\right)(x)-M\|\varphi\|-C} \leq$ $\lambda^{n+M} \nu\left(B_{n}(x ; \delta)\right) \leq e^{T_{n} \varphi(x)} s_{M}(2 \delta) e^{M\|\varphi\|+C} \forall x \in X, \forall n \geq 1$. Hence (ii) holds with $D_{\delta}=s_{M}(2 \delta) e^{M\|\varphi\|+C} \lambda^{-M}$.

Now suppose (ii) holds and we wish to prove (i). For sufficiently small $\delta \frac{\nu\left(B_{n}(x ; 2 \delta)\right)}{\nu\left(B_{n}(x ; \delta)\right)}$ $\leq D_{2 \delta} D_{\delta} \forall x \in X, \forall n \geq 1$. Let $d\left(T^{i} x, T^{i} x^{\prime}\right) \leq \delta_{/ 2}$ for $0 \leq i \leq n-1$. Then $x \in B_{n}\left(x^{\prime} ; \delta\right)$ so $B_{n}(x ; \delta) \subset B_{n}\left(x^{\prime} ; 2 \delta\right)$ and

$$
e^{T_{n} \varphi(x)-T_{n} \varphi\left(x^{\prime}\right)} \leq D_{\delta}^{2} \frac{\nu\left(B_{n}(x ; \delta)\right)}{\nu\left(B_{n}\left(x^{\prime} ; \delta\right)\right)} \leq D_{\delta}^{2} \frac{\nu\left(B_{n}\left(x^{\prime} ; 2 \delta\right)\right)}{\nu\left(B_{n}\left(x^{\prime} ; \delta\right)\right)} \leq D_{2 \delta} D_{\delta}^{3} .
$$

Therefore $\varphi$ satisfies Bowen's condition.

\section{3. $g$-MEASURES}

Let $T: X \rightarrow X$ be a positively expansive local homeomorphism satisfying the weak specification condition. Let $g: X \rightarrow(0,1)$ be continuous and satisfy $\sum_{y \in T^{-1} x} g(y)=1 \forall x \in X$. Such a function is called a $g$-function for $T$, and assigns, in a continuous way, a probability distribution to each of the finite sets $T^{-1} x$. We have $\left(\mathcal{L}_{\log g} f\right)(x)=\sum_{y \in T^{-1} x} g(y) f(y)$ and $\mathcal{L}_{\log g} 1=1$ so that $\left(P_{\log g}^{(n)} f\right)(x)=$ $\sum_{z \in T^{-n} T^{n} x} g(z) \cdots g\left(T^{n-1} z\right) f(z)$.

Hence $P_{\log g}^{(n)}=U_{T}^{n} \mathcal{L}_{\log g}^{(n)}$. By the Schauder-Tychanoff fixed point theorem there is always at least one $\mu \in M(X)$ with $\mathcal{L}_{\log g}^{*} \mu=\mu$. Every $\mu \in M(X)$ with $\mathcal{L}_{\log g}^{*} \mu=\mu$ is $T$-invariant, since if $f \in C(X ; \mathbb{R}) \int f \circ T d \mu=\int \mathcal{L}_{\log g}(f \circ T) d \mu=\int f d \mu$. In fact, using the notation of the previous section, we have, if $G_{g}=\left\{\mu \in M(X) \mid \mathcal{L}_{\log g}^{*} \mu=\right.$ $\mu\}$ is the set of $g$-measures:

\section{Theorem 3.1.}

$$
G_{g}=K_{\log g} \cap M(X, T)=K_{\log g}^{(1)} \cap M(X, T) .
$$

Proof. We have $G_{g} \subset K_{\log g}$ by Corollary 2.3 and $G_{g} \subset M(X, T)$ by the above. Hence $G_{g} \subset K_{\log g} \cap M(X, T) \subset K_{\log g}^{(1)} \cap M(X, T)$. However, if $\mu \in K_{\log g}^{(1)} \cap M(X, T)$ then $\mu \in G_{g}$. 
From Theorem 2.9 we know that $K_{\log g}$ has only one member iff $\forall f \in C(X ; \mathbb{R}) \exists c(f) \in \mathbb{R}$ with $\mathcal{L}_{\log g}^{n} f \rightrightarrows c(f)$. One can easily show that there is a unique $g$-measure iff $\forall f \in C(X ; \mathbb{R}) \exists c(f) \in \mathbb{R}$ with $\frac{1}{n} \sum_{i=0}^{n-1} \mathcal{L}_{\log g}^{i} f=c(f)$. Bramson and Kalikow have given examples of $g$-functions without unique $g$-measures ([B-K1]).

Our Theorem 2.16 gives the following result, which was proved by Fan in the case of topologically mixing subshifts of finite type $([\mathrm{F}])$.

Theorem 3.2. Let $T: X \rightarrow X$ be a positively expansive local homeomorphism with the weak specification condition. Let $g: X \rightarrow(0,1)$ be continuous and $\forall x \in$ $X \sum_{y \in T^{-1} x} g(y)=1$. Let $\log g$ satisfy Bowen's condition (i.e. $\exists \delta>0$ and $C>0$ such that $d\left(T^{i} x, T^{i} x^{\prime}\right) \leq \delta, 0 \leq i \leq n-1$ implies $\left.\frac{g(x) g(T x) \cdots g\left(T^{n-1} x\right)}{g\left(x^{\prime}\right) g\left(T x^{\prime}\right) \cdots g\left(T^{n-1} x^{\prime}\right)} \leq e^{C}\right)$. Then $\forall f \in C(X ; \mathbb{R}) \mathcal{L}_{\log g}^{n} f \Rightarrow$ constant. The set $K_{\log g}$ has only one member, in particular, there is a unique $g$-measure $\mu$. Also, $\mu$ is exact.

So for the collection of functions of the form $\log (g)$ Bowen's assumption implies the conclusion of Ruelle's theorem.

There are examples of $g$ 's with $\mathcal{L}_{\log g}^{n} f=$ constant but $\log g$ does not satisfy Bowen's condition $([\underline{\mathrm{H}}])$. Such an example is the following where $a \in(0,1)$.

On the space $X=\{0,1\}^{Z^{+}}$let

$$
g(x)= \begin{cases}a\left(\frac{n+1}{n+2}\right)^{3} & \text { if }\left(x_{0}, \ldots, x_{n+1}\right)=(1,1, \ldots, 1,1,0), n \geq 0 \\ 1-a\left(\frac{n+1}{n+2}\right)^{3} & \text { if }\left(x_{0}, \ldots, x_{n+1}\right)=(0,1,1, \ldots, 1,1,0), n \geq 0 \\ a & \text { if } x_{i}=1 \quad \forall i \geq 0 \\ 1-a & \text { if } x_{0}=0 \text { and } x_{i}=1 \quad \forall i \geq 1 .\end{cases}
$$

Theorem 2.18 gives the following

Theorem 3.3. Let $T: X \rightarrow X$ be a positively expansive local homeomorphism satisfying the weak specification condition. For a g-function $g: X \rightarrow(0,1)$ the following are equivalent:

(i) $\log g$ satisfies Bowen's condition.

(ii) $\exists \mu \in M(X, T)$ with the property that for every sufficiently small $\delta>0 \exists D_{\delta}>$ 1 with

$$
D_{\delta}^{-1} \leq \frac{\mu\left(B_{n}(x ; \delta)\right)}{g(x) \cdots g\left(T^{n-1} x\right)} \leq D_{\delta} \quad \forall x \in X, \quad \forall n \geq 1 .
$$

(iii) $\exists \mu \in M(X, T)$ such that for some expansive constants $\delta_{0} \exists D_{\delta_{0}}>1$ with

$$
D_{\delta_{0}}^{-1} \leq \frac{\mu\left(B_{n}\left(x ; \delta_{0}\right)\right)}{g(x) \cdots g\left(T^{n-1} x\right)} \leq D_{\delta_{0}} \quad \forall x \in X, \quad \forall n \geq 0 .
$$

Proof. In the proof of Theorem 2.18 we showed a measure satisfies the property in (ii) iff it satisfies the property in (iii). If (i) holds and we choose $\mu$ with $\mathcal{L}_{\log g}^{*} \mu=\mu$, then the proof of Theorem 2.18, with $\nu=\mu, \lambda=1$, shows $\mu$ satisfies (ii). We get (iii) implies (i) by Theorem 2.18 .

Corollary 4.7 will give more information. 


\section{Equilibrium states}

In this section we want to study equilibrium states and study the convergence of $\frac{\mathcal{L}_{\varphi}^{n} f}{\lambda^{n}}$ as $n \rightarrow \infty$. We use a method involving measurable $g$-functions.

Let $T: X \rightarrow X$ be a positively expansive local homeomorphism with the weak specification property. If $\varphi \in C(X ; \mathbb{R})$ satisfies Bowen's condition, Theorem 2.16 gives a unique $\nu \in M(X)$ and unique $\lambda>0$ with $\mathcal{L}_{\varphi}^{*} \nu=\lambda \nu, \lambda$ equals $e^{P(T, \varphi)}$ and $\nu$ is tail-trivial. Also $\exists D>1$ with $D^{-1} \leq \frac{\mathcal{L}_{\varphi}^{n} 1(x)}{\lambda^{n}} \leq D \forall x \in X, \forall n \geq 1$.

The following gives information about solving the equation $\mathcal{L}_{\varphi} h=\lambda h$ when $\varphi \in C(X ; \mathbb{R})$.

Theorem 4.1. Let $T: X \rightarrow X$ be a positively expansive local homeomorphism satisfying the weak specification condition. For $\varphi \in C(X ; \mathbb{R})$ and $\lambda>0$ the following statements are equivalent:

(i) $\exists D>1$ with $D^{-1} \leq \frac{\mathcal{L}_{\varphi}^{n} 1(x)}{\lambda^{n}} \leq D \forall x \in X, \forall n \geq 1$.

(ii) $\exists$ measurable $h: X \rightarrow\left[d_{1}, d_{2}\right] \subset(0, \infty)$ with $\mathcal{L}_{\varphi} h=\lambda h$.

(iii) $\exists h: X \rightarrow\left[d_{1}, d_{2}\right] \subset(0, \infty)$ with $\mathcal{L}_{\varphi} h=\lambda h$.

Proof. Assume (i) holds. Let $h_{1}(x)=\limsup _{n \rightarrow \infty} \frac{\mathcal{L}_{\varphi}^{n} 1(x)}{\lambda^{n}}$. Then $h_{1}: X \rightarrow\left[D^{-1}, D\right]$ is measurable and one readily gets $\lambda h_{1}(x) \leq\left(\mathcal{L}_{\varphi} h_{1}\right)(x)$. To get $h$ with $\mathcal{L}_{\varphi} h=\lambda h$ everywhere we can proceed as follows.

We have $\frac{\left(\mathcal{L}_{\varphi}^{n} h_{1}\right)}{\lambda^{n}} \leq D \frac{\mathcal{L}_{\varphi}^{n} 1(x)}{\lambda^{n}} \leq D^{2} \forall n \geq 1, \forall x \in X$ and since $D^{-1} \leq h_{1} \leq$ $\frac{\mathcal{L}_{\varphi} h_{1}}{\lambda} \leq \frac{\mathcal{L}_{\varphi}^{2} h_{1}}{\lambda^{2}} \leq \ldots$, if we let $h(x)=\lim _{n \rightarrow \infty} \frac{\left(\mathcal{L}_{\varphi}^{n} h_{1}\right)(x)}{\lambda^{n}}=\sup _{n} \frac{\left(\mathcal{L}_{\varphi}^{n} h_{1}\right)(x)}{\lambda^{n}}$, then $h$ is measurable, $h: X \rightarrow\left[D^{-1}, D^{2}\right]$, and $\mathcal{L}_{\varphi} h=\lambda h$ everywhere. Hence (ii) holds. Clearly (ii) implies (iii). It remains to show (iii) implies (i). From $\left(\mathcal{L}_{\varphi}^{n} h\right)(x)=$ $\lambda^{n} h(x)$ we have $d_{1} \mathcal{L}_{\varphi}^{n} 1(x) \leq \lambda^{n} d_{2}$ so that $\frac{\mathcal{L}_{\varphi}^{n} 1(x)}{\lambda^{n}} \leq \frac{d_{2}}{d_{1}}$. Similarly, $d_{2} \mathcal{L}_{\varphi}^{n} 1(x) \geq \lambda^{n} d_{1}$ so $\frac{d_{1}}{d_{2}} \leq \frac{\mathcal{L}_{\varphi}^{n} 1(x)}{\lambda^{n}}$.

The discussion before Theorem 4.1 gives

Corollary 4.2. If $\varphi$ satisfies Bowen's condition and $\lambda=e^{P(T, \varphi)}$, there exists a measurable $h: X \rightarrow\left[d_{1}, d_{2}\right] \subset(0, \infty)$ with $\mathcal{L}_{\varphi} h=\lambda h$ everywhere.

From now on we assume $\varphi \in C(X ; \mathbb{R})$ satisfies Bowen's condition and that $\nu \in M(X), \lambda>0, h: X \rightarrow\left[d_{1}, d_{2}\right] \subset(0, \infty)$ are so that $\mathcal{L}_{\varphi}^{*} \nu=\lambda \nu, h$ is measurable, $\mathcal{L}_{\varphi} h=\lambda h$ and $\nu(h)=1$. The last condition can be attained by replacing $h$ in Corollary 4.2 by $\frac{h}{\nu(h)}$. If we want to emphasize dependence on $\varphi$ we use $\nu_{\varphi}, \lambda_{\varphi}, h_{\varphi}$.

Theorem 4.3. Let $\varphi$ satisfy Bowen's condition and let $\nu, \lambda, h$ be as above. Let $\mu=h \cdot \nu$. Then $\mu \in M(X, T)$ and $T$ is an exact endomorphism with respect to $\mu$. If $h^{\prime}: X \rightarrow(0, \infty)$ is measurable with $\nu\left(h^{\prime}\right)=1$ and $\mathcal{L}_{\varphi} h^{\prime}=\lambda h^{\prime}$ a.e. $(\nu)$, then $h^{\prime}=h$ a.e. $(\nu)$.

Proof. If $f \in C(X ; \mathbb{R})$, then $\int f \circ T \cdot h d \nu=\lambda^{-1} \int \mathcal{L}_{\varphi}(f \circ T \cdot h) d \nu=\lambda^{-1} \int f \cdot \mathcal{L}_{\varphi} h d \nu=$ $\int f \cdot h d \nu$, so $\mu$ is $T$-invariant. The measures $\nu$ and $h \cdot \nu$ are equivalent so $\mu$ is also tail-trivial, and hence $T$ is exact with respect to $\mu$. If $h^{\prime}$ is as in the statement of the theorem, then $\mu^{\prime}=h^{\prime} \cdot \nu$ is also in $M(X, T)$ and is tail-trivial and so both $\mu$ and $\mu^{\prime}$ are ergodic. Since $\mu^{\prime} \ll \mu$ we must have $\mu^{\prime}=\mu$ and hence $h^{\prime}=h$ a.e. $(\nu)$.

From now on we use $\mu$ for $h \cdot \nu$, and write it $\mu_{\varphi}$ if we wish to emphasize its relationship to $\varphi$. Note $L_{\nu}^{p}(X)=L_{\mu}^{p}(x) \forall p \geq 1$. Also, let $g=\frac{e^{\varphi} h}{\lambda \cdot h \circ T}$. Then 
$g$ is measurable, $g: X \rightarrow[a, b] \subset(0,1)$ and $\forall x \in X \sum_{y \in T^{-1} x} g(y)=1$. Let $\mathcal{L}_{\log g}: L(X ; \mathbb{R}) \rightarrow L(X ; \mathbb{R})$ be defined by $\left(\mathcal{L}_{\log g} f\right)(x)=\sum_{y \in T^{-1} x} g(y) f(y)$. We have for $n \geq 1$

$$
\left(\mathcal{L}_{\log g}^{n} f\right)(x)=\frac{1}{\lambda^{n} h(x)}\left(\mathcal{L}_{\varphi}^{n}(h \cdot f)\right)(x) .
$$

From Corollary 2.8 $\int \mathcal{L}_{\varphi} f d \nu=\lambda \int f d \nu \forall f \in L_{\nu}^{1}(X)$ so we have

$$
\int \mathcal{L}_{\log g} f d \mu=\int f d \mu \quad \forall f \in L_{\nu}^{1}(X)=L_{\mu}^{1}(X) .
$$

We have the following version of a result of Ledrappier $([\underline{\mathrm{L}})$. We write $\mathcal{B}$ instead of $\mathcal{B}(X)$ and if $\tau \in M(X, T)$, then $h_{\tau}(T)$ denotes the entropy of the measurepreserving transformation $T:(X, \mathcal{B}, \tau) \rightarrow(X, \mathcal{B}, \tau)$.

Lemma 4.4. Let $T: X \rightarrow X$ be a positively expansive continuous surjection and let $g: X \rightarrow[a, b] \subset(0,1)$ be measurable and satisfy $\sum_{y \in T^{-1} x} g(y)=1 \forall x \in X$.

We have $h_{\tau}(T)+\int \log g d \tau \leq 0 \forall \tau \in M(X, T)$. For $\sigma \in M(X, T)$ the following statements are pairwise equivalent:

(i) $\int \mathcal{L}_{\log g} f d \sigma=\int f d \sigma \forall f \in L_{\sigma}^{1}(X)$.

(ii) $\sigma \in M(X, T)$ and $\forall f \in L_{\sigma}^{1}(X) E_{\sigma}\left(f_{/ T^{-1} \mathcal{B}}\right)(x)=\sum_{y \in T^{-1} T x} g(y) f(y)$ a.e. $(\sigma)$.

(iii) $\sigma \in M(X, T)$ and $h_{\sigma}(T)+\int \log g d \sigma=0$.

Proof. We write $\mathcal{L}$ instead of $\mathcal{L}_{\log g}$.

Since $T$ is positively expansive, every finite partition of $X$ into sets of sufficiently small diameter is a one-sided generator. Hence $h_{\tau}(T)=H_{\tau}\left(\mathcal{B}_{/ T^{-1} \mathcal{B}}\right) \forall \tau \in$ $M(X, T)$. If $g_{\tau}: X \rightarrow[0,1]$ is defined a.e. $(\tau)$ by

$$
E_{\tau}\left(f_{/ T^{-1} \mathcal{B}}\right)(x)=\sum_{y \in T^{-1} T x} g_{\tau}(y) f(y), f \in L_{\tau}^{1},
$$

then

$$
\begin{aligned}
h_{\tau}(T) & =H_{\tau}\left(\mathcal{B}_{/ T^{-1} \mathcal{B}}\right)=-\int \sum_{y \in T^{-1} T x} g_{\tau}(y) \log g_{\tau}(y) d \tau(x) \\
& =-\int \log g_{\tau} d \tau .
\end{aligned}
$$

Hence

$$
\begin{aligned}
h_{\tau}(T)+\int \log g d \tau & =\int \log g / g_{\tau} d \tau \\
& \leq \int\left(\frac{g}{g_{\tau}}-1\right) d \tau \\
& =\int \sum_{y \in T^{-1} T x} g_{\tau}(y)\left(\frac{g(y)}{g_{\tau}(h)}-1\right) d \tau(x) \\
& =0 \text { since } \sum_{y \in T^{-1} T x} g(y)=1 \quad \forall x \in X .
\end{aligned}
$$

Equality holds here iff $\log g / g_{\tau}=g / g_{\tau}-1$ a.e. $(\tau)$ i.e. $g=g_{\tau}$ a.e. $(\tau)$. Hence $h_{\tau}(T)+\int \log g d \tau \leq 0 \forall \tau \in M(X, T)$. 
(i) $\Rightarrow$ (ii) Let $f \in L_{\sigma}^{1}$. Then $\int f \circ T d \sigma=\int \mathcal{L}(f \circ T) d \sigma=\int f d \sigma$ so $\sigma \in M(X, T)$. Also, if $B \in \mathcal{B}$

$$
\begin{aligned}
\int_{T^{-1} B} f d \sigma & =\int f \cdot \chi_{B} \circ T d \sigma=\int \mathcal{L}\left(f \cdot \chi_{B} \circ T\right) d \sigma \\
& =\int\left(\mathcal{L}\left(f \cdot \chi_{B} \circ T\right)\right) \circ T d \sigma=\int_{T^{-1} B} \sum_{y \in T^{-1} T x} g(y) f(y) d \sigma(x) .
\end{aligned}
$$

(ii) $\Rightarrow$ (iii) By the above if (ii) holds, then $g=g_{\sigma}$ and (iii) holds.

(iii) $\Rightarrow$ (i) From the above proof we know that for $\tau \in M(X, T) h_{\tau}(T)+\int \log g d \tau$ $=0$ iff $g_{\tau}=g$ a.e. $(\tau)$. So if (iii) holds, then $g_{\sigma}=g$ a.e. $(\sigma)$. Hence for $f \in L_{\sigma}^{1}$

$$
\int \mathcal{L} f d \sigma=\int(\mathcal{L} f) \circ T d \sigma=\int \sum_{y \in T^{-1} T x} g(y) f(y) d \sigma(x)=\int f d \sigma .
$$

Since our measure $\mu=h \cdot \nu$ satisfies statement (i) of Lemma 4.4, it also satisfies (ii) and (iii). We use this to show $\mu$ is the unique equilibrium state of $\varphi$. Recall that $\mu$ is an equilibrium state of $\varphi$ if $\forall \tau \in M(X, T) h_{\tau}(T)+\int \varphi d \tau \leq h_{\mu}(T)+\int \varphi d \mu$; equivalently if $h_{\mu}(T)+\int \varphi d \mu=P(T, \varphi)$.

Theorem 4.5. Let $T: X \rightarrow X$ be a positively expansive local homeomorphism satisfying the weak specification condition. Let $\varphi \in C(X ; \mathbb{R})$ satisfy Bowen's condition. Then $\varphi$ has a unique equilibrium state and this state is $\mu=h \cdot \nu$. With respect to $\mu T$ is exact.

Proof. Since $\log \lambda=P(T, \varphi)$, we know $\sigma \in M(X, T)$ is an equilibrium state for $\varphi$ iff $h_{\sigma}(T)+\int \varphi d \sigma=\log \lambda$. Since $\varphi=\log g+\log \lambda+\log h \circ T-\log h$ this is equivalent to $h_{\sigma}(T)+\int \log g d \sigma=0$. By Lemma 4.4 this is equivalent to $\int \mathcal{L}_{\log g} f d \sigma=$ $\int f d \sigma \forall f \in L_{\sigma}^{1}(X)$, which is equivalent to $\int \mathcal{L}_{\varphi} f d \tau=\lambda \int f d \tau \forall f \in L_{\tau}^{1}(X)$, where $\tau=\frac{1}{h} \sigma$. This gives $\mathcal{L}_{\varphi}^{*} \tau=\lambda \tau$ and we know, by Theorem 2.16 (i), that $\nu$ is the only probability measure that satisfies this. Hence $\mu=h \cdot \nu$ is an equilibrium state for $\varphi$. If $\sigma$ is an equilibrium state for $\varphi$, then $\sigma=c \cdot h \cdot \nu$ for some $c>0$. Since $\sigma$ and $h \cdot \nu$ are both probability measures we get $c=1$, and $\mu=h \cdot \nu$ is the only equilibrium state for $\varphi$. Exactness was proved in Theorem 4.3.

Theorem 4.6. Let $T: X \rightarrow X$ be a positively expansive local homeomorphism satisfying the weak specification condition. Let $\varphi \in C(X ; \mathbb{R})$ satisfy Bowen's condition, and let $\lambda=e^{P(T, \varphi)}$. The following three statements about $\sigma \in M(X, T)$ are pairwise equivalent:

(i) $\sigma$ is the unique equilibrium state $\mu=h \cdot \nu$.

(ii) For every sufficiently small $\delta>0 \exists E_{\delta}>1$ with

$$
E_{\delta}^{-1} \leq \frac{\sigma\left(B_{n}(x ; \delta)\right)}{\lambda^{-n} e^{\left(T_{n} \varphi\right)(x)}} \leq E_{\delta} \quad \forall x \in X, \quad \forall n \geq 1 .
$$

(iii) For some expansive constant $\delta_{0} \exists E_{\delta_{0}}>1$ with

$$
E_{\delta_{0}}^{-1} \leq \frac{\sigma\left(B_{n}\left(x ; \delta_{0}\right)\right)}{\lambda^{-n} e^{\left(T_{n} \varphi\right)(x)}} \leq E_{\delta_{0}} \quad \forall x \in X, \quad \forall n \geq 1
$$


Proof. Let $\nu, \lambda, h$ be associated to $\varphi$ as usual.

Assume (i). We have $0<d^{-1} \leq h \leq d$ for some $d>1$. By the proof of Theorem 2.18 we know $\lambda, \nu$ satisfy statement (ii) of Theorem 2.18 so we have

$$
E_{\delta}^{-1} \leq \frac{\mu\left(B_{n}(x ; \delta)\right)}{\lambda^{-n} e^{\left(T_{n} \varphi\right)(x)}} \leq E_{\delta} \quad \forall x \in X, \quad \forall n \geq 1
$$

if $E_{\delta}=d D_{\delta}$. Hence (ii) holds.

Clearly (ii) implies (iii). Assume (iii) holds and we prove (i). We have

$$
-\frac{1}{n} \log \sigma\left(B_{n}\left(x ; \delta_{0}\right)\right)+\frac{1}{n}\left(T_{n} \varphi\right)(x) \rightarrow \log \lambda=P(T, \varphi) .
$$

Integrating with respect to $\sigma$, and using the Brin-Katok local entropy formula ([B-K2]), gives $h_{\sigma}(T)+\int \varphi d \sigma=P(T, \varphi)$. This says $\sigma$ is an equilibrium state for $\varphi$ and so must equal the unique one $\mu$.

We have the following special case.

Corollary 4.7. Let $T: X \rightarrow X$ be a positively expansive local homeomorphism satisfying the weak specification condition. Let $g \in C(X ; \mathbb{R})$ be a g-function so that $\log g$ satisfies Bowen's condition. Then the unique $g$-measure is the only member $\mu$ of $M(X, T)$ with either of the following properties:

(i) for every sufficiently small $\delta>0 \exists E_{\delta}>1$ with

$$
E_{\delta}^{-1} \leq \frac{\mu\left(B_{n}(x ; \delta)\right)}{g(x) g(T x) \cdots g\left(T^{n-1} x\right)} \leq E_{\delta} \quad \forall x \in X, \quad \forall n \geq 1 ;
$$

(ii) for some expansive constant $\delta_{0} \exists E_{\delta_{0}}>1$ with

$$
E_{\delta_{0}}^{-1} \leq \frac{\mu\left(B_{n}\left(x ; \delta_{0}\right)\right)}{g(x) g(T x) \cdots g\left(T^{n-1} x\right)} \leq E_{\delta_{0}} \quad \forall x \in X, \quad \forall n \geq 1 .
$$

The following generalizes Theorem 3.3

Theorem 4.8. Let $T: X \rightarrow X$ be a positively expansive local homeomorphism with the weak specification condition. The following statements about $\varphi \in C(X ; \mathbb{R})$ are pairwise equivalent:

(i) $\varphi$ satisfies Bowen's condition.

(ii) $\exists \mu \in M(X, T)$ and $\lambda>0$ with the property that for all sufficiently small $\delta>0 \exists E_{\delta}>1$ with

$$
E_{\delta}^{-1} \leq \frac{\mu\left(B_{n}(x ; \delta)\right)}{\lambda^{-n} e^{\left(T_{n} \varphi\right)(x)}} \leq E_{\delta} \quad \forall x \in X, \quad \forall n \geq 1 .
$$

(iii) $\exists \mu \in M(X, T)$ and $\lambda>0$ with the property that for some expansive constant $\delta_{0} \exists E_{\delta_{0}}>1$ with

$$
E_{\delta_{0}}^{-1} \leq \frac{\mu\left(B_{n}\left(x ; \delta_{0}\right)\right)}{\lambda^{-n} e^{\left(T_{n} \varphi\right)(x)}} \leq E_{\delta_{0}} \quad \forall x \in X, \quad \forall n \geq 1 .
$$

Proof. We get (i) $\Rightarrow$ (ii) by Theorem 4.6. Clearly (i) $\Rightarrow$ (iii), and we get (iii) $\Rightarrow$ (i) by Theorem 2.8 .

We can now make some deductions about equilibrium states using a result of Quas QQ. The result of Quas says, in our context, that if $T: X \rightarrow X$ is a positively expansive local homeomorphism satisfying the weak specification property and $\mu$ is an ergodic member of $M(X, T)$ with full support, then if $F \in L_{\mu}^{\infty}(X)$ satisfies $F \circ T-F=f \in C(X ; \mathbb{R})$ a.e. there is $G \in C(X ; \mathbb{R})$ with $G \circ T-G=f$ everywhere. 
We shall let $\varphi \in C(X ; \mathbb{R})$ satisfy Bowen's condition and let $\lambda, \nu, h$ be so that $\mathcal{L}_{\varphi}^{*} \nu=$ $\lambda \nu, \mathcal{L}_{\varphi} h=\lambda h, \nu(h)=1$. We know, by Theorem 2.8, that $\nu\left(B_{n}(x, \delta)\right)>0 \forall x \in X$, $\forall n \geq 1$ so that if $U \neq \varnothing$ is open, $\nu(U)>0$ because $B_{n}(x, \delta) \subset U$ for some $n \geq 1$ and some $x$. Hence $\mu=h \cdot \nu$ is an ergodic $T$-invariant measure with full support.

Theorem 4.9. Let $T: X \rightarrow X$ be a positively expansive local homeomorphism satisfying the weak specification conditions. Let $\varphi_{1}, \varphi_{2} \in C(X ; \mathbb{R})$ both satisfy Bowen's condition. Then $\mu_{\varphi_{1}}=\mu_{\varphi_{2}}$ iff $\exists c \in \mathbb{R}$ and $f \in C(X ; \mathbb{R})$ with $\varphi_{1}-\varphi_{2}=c+f \circ T-f$.

Proof. Let $\mu_{\varphi_{1}}=\mu_{\varphi_{2}}=\mu$. Then $g_{\varphi_{1}}=g_{\varphi_{2}}$ a.e. $\mu$ so $\frac{e^{\varphi_{1}} h_{1}}{\lambda_{1} h_{1} \circ T}=\frac{e^{\varphi_{2} h_{2}}}{\lambda_{2} h_{2} \circ T}$ a.e. $(\mu)$ where the $\lambda_{i}, h_{i}$ correspond to $\varphi_{i}, i=1,2$. Hence $\varphi_{1}-\varphi_{2}=\log \left(\lambda_{1} / \lambda_{2}\right)+H \circ T-H$ a.e. $(\mu)$ where $H=\log h_{1}-\log h_{2}$. Since $H \in L_{\mu}^{\infty}(X)$, the result of Quas mentioned above implies $\exists f \in C(X ; \mathbb{R})$ with $\varphi_{1}-\varphi_{2}=\log \left(\lambda_{1} / \lambda_{2}\right)+f \circ T-f$ everywhere.

Conversely, if $\varphi_{1}-\varphi_{2}=c+f \circ T-f$ with $f \in C(X ; \mathbb{R})$, then $\varphi_{1}, \varphi_{2}$ have the same equilibrium states, since $\sigma\left(\varphi_{1}\right)=\sigma\left(\varphi_{2}\right)+c \forall \sigma \in M(X, T)$, so $\mu_{\varphi_{1}}=\mu_{\varphi_{2}}$ by Theorem 4.5.

This result was known under the stronger assumption of Theorem 1.3 ([W1], p. 134).

Theorem 4.10. Let $T: X \rightarrow X$ be a positively expansive local homeomorphism satisfying the weak specification condition. Let $\varphi \in C(X ; \mathbb{R})$ satisfy Bowen's condition. The equivalent statements (i) to (vi) of Corollary 2.17 are each equivalent to: (vii) the unique equilibrium state $\mu$ of $\varphi$ is a g-measure for a continuous $g: X \rightarrow(0,1)$.

Proof. we show (vii) is equivalent to the existence of $h \in C(X ; \mathbb{R})$ with $h>0$ and $\mathcal{L}_{\varphi} h=\lambda h$, which is statement (ii) of Corollary 2.17. If such a $h$ exists, then $g=\frac{e^{\varphi} h}{\lambda \cdot h \circ T}$ is continuous and $\mu$ is a $g$-measure for this $g$, so that (vii) holds.

Now assume (vii) holds. If $\mu$ is a $g$-measure for a continuous $g$, then $g=\frac{e^{\varphi} h}{\lambda \cdot h \circ T}$ a.e. $(\mu)$ where $\mathcal{L}_{\varphi} h=\lambda h$ and $h: X \rightarrow\left[d_{1}, d_{2}\right] \subset(0, \infty)$ is measurable. This says $\varphi-\log g-\log \lambda=\log h \circ T-\log h$ a.e. $(\mu)$, and since the left-hand side is continuous and $\log h \in L_{\mu}^{\infty}(X)$ we can use Quas' result to obtain $H \in C(X ; \mathbb{R})$ with $\varphi-\log g-\log \lambda=H \circ T-H$ everywhere. Since $\sum_{y \in T^{-1} x} g(y)=1 \forall x \in X$ we have $\mathcal{L}_{\varphi} e^{H}=\lambda e^{H}$.

We now turn to the convergence of $\frac{\mathcal{L}_{\varphi}^{n} f}{\lambda^{n}}$.

In the following we use $\|\cdot\|_{p}$ to denote the norm in $L_{\nu}^{p}(X)$. The following result was obtained by Ruelle by another method $([\overline{\mathrm{Ru}}])$.

Theorem 4.11. Let $T: X \rightarrow X$ be a positively expansive local homeomorphism satisfying the weak specification condition. Let $\varphi \in C(X ; \mathbb{R})$ satisfy Bowen's condition. Let $\lambda>0$ and $v \in M(X)$ satisfy $\mathcal{L}_{\varphi}^{*} \nu=\lambda \nu$ and let $h: X \rightarrow\left[d_{1}, d_{2}\right] \subset(0, \infty)$ be measurable with $\mathcal{L}_{\varphi} h=\lambda h$ and $\nu(h)=1$. For every $p \geq 1$ and every $f \in L_{\nu}^{p}(X)$

$$
\left\|\frac{\left(\mathcal{L}_{\varphi}^{n} f\right)(x)}{\lambda^{n}}-h(x) \nu(f)\right\|_{p} \rightarrow 0 \text { as } n \rightarrow \infty
$$

Proof.

$$
\begin{aligned}
\frac{\mathcal{L}_{\varphi}^{n} f(x)}{\lambda^{n}}-h(x) \nu(f) & =\left[\mathcal{L}_{\log g}^{n}(f / h)(x)-\nu(f)\right] h(x) \\
& =\left[\mathcal{L}_{\log g}^{n}(f / h)(x)-\mu(f / h)\right] h(x)
\end{aligned}
$$


Since $0<d_{1} \leq h \leq d_{2}$, we have $f \in L_{\nu}^{p}(X)$ iff $f / h \in L_{\mu}^{p}(X)$, and it suffices to show $\left\|\left(\mathcal{L}_{\log g}^{n} F\right)(x)-\mu(F)\right\|_{p} \rightarrow 0 \forall F \in L_{\mu}^{p}(X)$, where $\|\cdot\|_{p}$ now denotes the norm in $L_{\mu}^{p}(X)$. By the $L^{1}$ and $L^{p}, p>1$, convergence theorems ([P], pp. 231, 234) $E_{\mu}\left(F / T^{-n} \mathcal{B}(X)\right) \rightarrow E_{\mu}\left(F / \bigcap_{n=0}^{\infty} T^{-n} \mathcal{B}(X)\right)$ in $L_{\mu}^{p}(X)$. Since $\mu$ is tail-trivial, the limit is $\mu(F)$. Hence $\left\|\left(\mathcal{L}_{\log g}^{n} F\right)\left(T^{n} x\right)-\mu(F)\right\|_{p} \rightarrow 0$ so, since $\mu$ is $T$-invariant, $\left\|\left(\mathcal{L}_{\log g}^{n} F\right)(x)-\mu(F)\right\|_{p} \rightarrow 0$.

Corollary 4.12. Let $T: X \rightarrow X$ be a positively expansive local homeomorphism satisfying the weak specification condition. There is an increasing sequence of integers $n_{1}<n_{2}<n_{3} \leq \cdots$ and a set $B \in \mathcal{B}(X)$ with $\mu(B)=1$, where $\mu$ is the unique equilibrium state of $\varphi$, such that $\forall f \in C(X ; \mathbb{R}) \forall x \in B$

$$
\frac{\left(\mathcal{L}_{\varphi}^{n_{i}} f\right)(x)}{\lambda^{n_{i}}} \rightarrow h(x) \nu(f)
$$

Proof. By Theorem 4.11 with $f=1$ we get $\left\{n_{i}\right\}$ and $B \in \mathcal{B}(X)$ with $\nu(B)=1$ so that $\frac{\left(\mathcal{L}_{\varphi}^{n_{i}} 1\right)(x)}{\lambda^{n_{i}}} \rightarrow h(x) \forall x \in B$. Combining this with Theorem 2.16 (iii) gives the result.

\section{Properties of the Density FUnCtion $h$}

Let $T: X \rightarrow X$ be a positively expansive local homeomorphism satisfying the weak specification property, and let $\varphi \in C(x ; \mathbb{R})$ satisfy Bowen's condition. From Corollary 4.2 we know there is a measurable $h: X \rightarrow\left[d_{1}, d_{2}\right] \subset(0, \infty)$ with $\mathcal{L}_{\varphi} h=$ $\lambda h$ and we can normalize so that $\nu(h)=1$.

Let $\bar{h}: X \rightarrow\left[d_{1}, d_{2}\right]$ be defined by $\bar{h}(x)=\lim _{\delta \rightarrow 0} \sup \{h(y) \mid d(x, y)<\delta\}$. Then $\bar{h}$ is upper semi-continuous and $\lambda \bar{h} \leq \mathcal{L}_{\varphi} \bar{h}$. Since both sides have the same integral with respect to $\nu$, we get $\lambda \bar{h}=\mathcal{L}_{\varphi} \bar{h}$ a.e. $(\nu)$. Similarly, if $\underline{h}: X \rightarrow\left[d_{1}, d_{2}\right]$ is defined by $\underline{h}(x)=\lim _{\delta \rightarrow 0} \inf \{h(y) \mid d(x, y)<\delta\}$, then $\underline{h}$ is lower semi-continuous and $\lambda \underline{h} \geq \mathcal{L}_{\varphi} \underline{h}$ so $\lambda \underline{h}=\mathcal{L}_{\varphi} \underline{h}$ a.e. $(\nu)$. We have $\underline{h} \leq h \leq \bar{h}$ so that $\nu(h) \leq 1 \leq \nu(\bar{h})$, and by Theorem $4.3 h=\frac{\bar{h}}{\nu(\bar{h})}=\frac{\underline{h}}{\nu(\underline{h})}$ a.e. $(\nu)$.

Theorem 5.1. We have $\frac{\underline{h}}{\nu(\underline{h})} \leq \frac{\bar{h}}{\nu(\bar{h})}$ everywhere. Also,

$$
\left\{x \in X \mid \frac{\underline{h}(x)}{\nu(\underline{h})}=\frac{\bar{h}(x)}{\nu(\bar{h})}\right\} \subset\{x \in X \mid \underline{h} \text { and } \bar{h} \text { are continuous at } x\}
$$

and both sets are dense $G_{\delta}$ sets with full $\nu$-measure.

Proof. Let $c=\frac{\nu(\bar{h})}{\nu(\underline{h})} \geq 1$. Then $\bar{h}=c \underline{h}$ is upper semicontinuous so that $\{x \mid(\bar{h}=$ $c \underline{h})(x)<0\}$ is open and has $\nu$-measure zero. Hence this set is empty and $c \underline{h} \leq \bar{h}$ everywhere. We have

$$
\{x \in X \mid \underline{c h}(x)=\bar{h}(x)\}=\bigcap_{n=1}^{\infty}\left\{x \in X \mid(\bar{h}-c \underline{h})(x)<\frac{1}{n}\right\}
$$

so this set is a $G_{\delta}$ set, and since it has $\nu$-measure 1 it must be dense. Let $x$ be so that $c \underline{h}(x)=\bar{h}(x)$ and we want to show $\bar{h}$ and $\underline{h}$ are continuous at $x$. Let $\varepsilon>0$. Since $\overline{\bar{h}}$ is upper semicontinuous at $x$ and $\underline{h}$ is lower semicontinuous at $x \exists \delta>0$ so that $d(z, x)<\delta$ implies $\bar{h}(z)<\bar{h}(x)+\varepsilon$ and $\underline{h}(x)-\varepsilon<\underline{h}(z)$. Therefore if $d(z, x)<\delta$, then

$$
\bar{h}(x)-c \varepsilon=c(\underline{h}(x)-\varepsilon)<c \underline{h}(z) \leq \bar{h}(z)<\bar{h}(x)+\varepsilon
$$


so $|\bar{h}(z)-\bar{h}(x)|<c \varepsilon$. Also, $d(z, x)<\delta$ implies

$$
\underline{h}(z)=\left(1+\frac{1}{c}\right) \varepsilon<\frac{1}{c} \bar{h}(z)-\left(1+\frac{1}{c}\right) \varepsilon<\frac{1}{c} \bar{h}(x)-\varepsilon=\underline{h}(x)-\varepsilon<\underline{h}(z)
$$

so that $|\underline{h}(z)-\underline{h}(x)|<\varepsilon$.

Corollary 5.2. If $\inf _{x} \frac{\bar{h}(x)}{h(x)}=1$ (in particular, if $h$ is continuous at one point), then $\nu(\underline{h})=\nu(\bar{h})$ and $\nu(\{x \in X \mid h$ is continuous at $x\})=1$.

Proof. We have $1 \leq \frac{\nu(\bar{h})}{\nu(\underline{h})} \leq \frac{\bar{h}(x)}{\underline{h}(x)} \forall x \in X$ so the assumption implies $\nu(\bar{h})=\nu(\underline{h})$. By Theorem 5.1$]\{x \in X \mid \underline{h}(x)=\bar{h}(x)\} \subset\{x \in X \mid \underline{h}$ and $\bar{h}$ are continuous at $x\}$ so if $\underline{h}(x)=\bar{h}(x)$, then using $\underline{h} \leq h \leq \bar{h}$ gives $\underline{h}(z)-\underline{h}(x) \leq h(z)-h(x) \leq \bar{h}(z)-\bar{h}(x)$ and hence $h$ is continuous at $x$.

Therefore the density $\frac{d \mu}{d \nu}$ has a version $\frac{\bar{h}}{\nu(\bar{h})}$ which is upper semicontinuous and is continuous at $\nu$-almost every point, and a version $\frac{h}{\nu(\underline{h})}$ which is lower semicontinuous and is continuous at $\nu$-almost every point.

The author does not know if there exists a continuous density $h$ when $\varphi$ satisfies Bowen's condition.

\section{REFERENCES}

[A-H] N. Aoku and K. Hiraide, Topological Theory of Dynamical Systems, North-Holland, 1994. MR 95m:58095

[B1] R. Bowen, Some systems with unique equilibrium states, Math. Systems Theory 8 (1974), 193-202. MR 53:3257

[B2] R. Bowen, Equilibrium States and the Ergodic Theory of Anosov Diffeomorphisms, Lecture Notes in Math., vol. 470, Springer, Berlin, 1975. MR 56:1364

[B-K1] M. Bramson and S. Kalikow, Nonuniqueness in g-functions, Israel J. Math. 84 (1993), 153-160. MR 94h:28011

[B-K2] M. Brin and A. Katok, On local entropy, in Geometric Dynamics, Lecture Notes in Math., Vol. 1007, Springer, Berlin, 1983. MR 85c:58063

[F] Ai Hua Fan, A proof of the Ruelle operator theorem, Rev. Math. Phys. 7 (1995), 12411247. MR 97e:28034

[H] F. Hofbauer, Examples for the nonuniqueness of the equilibrium state, Trans. Amer. Math. Soc. 228 (1977), 223-241. MR 55:8312

[L] F. Ledrappier, Principe variationnel et systèmes dynamiques symboliques, Z. Wahr. und Verw. Gebiete 30 (1974), 185-202. MR 53:8384]

[P] K. Parthasarathy, Introduction to Probability and Measure, Macmillan, London, 1977. MR 58:31322a

[Q] A. Quas, Rigidity of continuous coboundaries, Bull. London Math. Soc. 29 (1997), 595600. MR 99c:28054

[Re] W. Reddy, Expanding maps on compact metric spaces, Topology Appl. 13 (1982), 327334. MR 83d:54070

[Ru] D. Ruelle, Thermodynamic formalism for maps satisfying positive expansiveness and specification, Nonlinearity 5 (1992), 1223-1236. MR 94a:58115

[W1] P. Walters, Invariant measures and equilibrium states for some mappings which expand distances, Trans. Amer. Math. Soc. 236 (1978), 121-153. MR 57:6371

[W2] P. Walters, An Introduction to Ergodic Theory, Graduate Texts in Math., vol. 79, Springer, Berlin, 1982. MR 84e:28017

University of Warwick, Mathematics Institute, Coventry CV4 7AL, England

E-mail address: pw@maths. warwick.ac.uk 